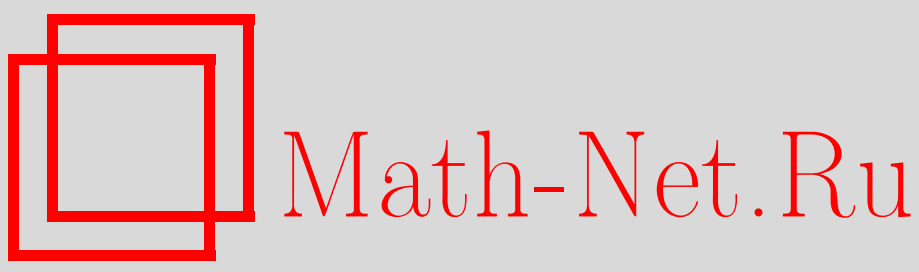

Л. С. Панкратов, В. А. Рыбалко, Асимптотический анализ модели двойной пористости с тонкими трещинами, Матем. сб., 2003, том 194, номер 1, 121-146

DOI: https://doi.org/10.4213/sm709

Использование Общероссийского математического портала Math-Net.Ru подразумевает, что вы прочитали и согласны с пользовательским соглашением http://www . mathnet.ru/rus/agreement

Параметры загрузки:

IP : 54.162 .127 .20

26 апреля 2023 г., 15:45:33 
УДК 517.946

\author{
Л. С. Панкратов, В. А. Рыбалко
}

\title{
Асимптотический анализ модели двойной пористости с тонкими трещинами
}

Рассматривается начально-краевая задача для параболического уравнения

$$
\Phi^{\varepsilon}(x) u_{t}^{\varepsilon}-\operatorname{div}\left(A^{\varepsilon}(x) \nabla u^{\varepsilon}\right)=f^{\varepsilon}(x), \quad x \in \Omega, \quad t>0,
$$

с разрывным тензором диффузии $A^{\varepsilon}(x)$. Предполагается, что этот тензор вырождается при $\varepsilon \rightarrow 0$ всюду в области $\Omega$ за исключением множества $\mathscr{F}^{(\varepsilon)}$ асимптотически малой меры. Показано, что поведение решений $u^{\varepsilon}$ при $\varepsilon \rightarrow 0$ описывается усредненной моделью с памятью.

Библиография: 25 названий.

\section{§1. Введение}

Задачи усреднения равномерно эллиптических дифференциальных операторов, а также соответствующих им эволюционных уравнений относятся к классическим и хорошо изученным вопросам теории усреднения (см., например, [1]-[5], где содержится обширная библиография). Другой интересный для приложений класс задач образуют модели, описываемые уравнениями с неравномерно эллиптическими дифференциальными операторами (под неравномерно әллиптическим оператором мы понимаем оператор с асимптотически вырождающейся на некотором множестве матрицей коэффициентов). Эти модели активно изучаются в настоящее время. Типичным примером такой модели служит хорошо известная модель двойной пористости, которая и является предметом настоящего исследования.

В работе мы рассматриваем начально-краевую задачу для параболического уравнения, описываюшего однофазное течение слабосжимаемой жидкости через пористую среду, состоящую из двух типов пород с контрастными транспортными свойствами. Такие среды нередко возникают в гидрологии и в нефтяной инженерии, когда пористые пласты состоят из пород с контрастными петрографическими характеристиками или пронизаны трещинами. Для описания таких сред обычно используется модель двойной пористости. В рамках этой модели пористая среда состоит из высокопроводящего связного множества трещин $\mathscr{F}^{(\varepsilon)}$ и множества $\mathscr{M}^{(\varepsilon)}$ (матрицы), образованного слабопроводящими непересекающимися включениями (блоками). Здесь $\varepsilon$ - характерный размер блоков. Задача состоит в макроскопическом (усредненном) описании течения жидкости в системе, когда параметр $\varepsilon$ мал.

Заметим, что модель течения жидкости через среду с двойной пористостью впервые была получена в [6] в предположении квазистационарности обмена между блоками и трещинами. В более общем случае, когда процесс обмена нельзя рассматривать как установившийся, макроскопическое описание этого процесса приводит к моделям с памятью (см., например, [7]-[11]).

Работа первого автора поддержана Украинским научно-техническим центром (грант № 1498).

(C) Л.С. Панкратов, В. А. РЫБАлко 2003 
В рамках классической модели двойной пористости предполагается, что толщина трещин имеет тот же порядок, что и размеры включений. Эта задача рассматривалась многими авторами. В дополнение к упомянутым выше работам отметим также статьи [12]-[16], в которых рассмотрены близкие вопросы. В настоящей работе в отличие от процитированной литературы мы предполагаем, что толщина трещин много меньше размеров блоков.

Перейдем к математической постановке задачи. Пусть $\Omega=\mathscr{F}^{(\varepsilon)} \cup \mathscr{M}^{(\varepsilon)} \cup \partial \mathscr{M}^{(\varepsilon)}$ - ограниченная область в $\mathbb{R}^{n}$ с кусочно-гладкой границей $\partial \Omega$, и пусть

$$
\text { meas } \mathscr{F}^{(\varepsilon)} \rightarrow 0, \quad \varepsilon \rightarrow 0 .
$$

Кроме того, в работе мы предполагаем, что множества $\mathscr{F}(\varepsilon)$ асимптотически распределены регулярно в области $\Omega$, т.е. сушествует постоянная $C>0$ такая, что для любого шара $V(y, r)=\{x:|x-y|<r\}$ с центром в точке $y \in \Omega$ и достаточно малых $\varepsilon>0, \varepsilon \leqslant \varepsilon_{0}(r)$,

$$
C^{-1} r^{n} \geqslant \mu^{\varepsilon} \operatorname{meas}\left(\mathscr{F}^{(\varepsilon)} \cap V(y, r)\right) \geqslant C r^{n},
$$

где

$$
\mu^{\varepsilon}=\frac{\operatorname{meas} \Omega}{\operatorname{meas} \mathscr{F}(\varepsilon)} .
$$

Рассмотрим следующую начально-краевую задачу:

$$
\begin{gathered}
\Phi^{\varepsilon}(x) \frac{\partial u^{\varepsilon}}{\partial t}-\operatorname{div}\left(A^{\varepsilon}(x) \nabla u^{\varepsilon}\right)=f^{\varepsilon}(x), \quad(x, t) \in \Omega_{T}, \\
\frac{\partial u^{\varepsilon}}{\partial \nu_{A^{\varepsilon}}}(x, t)=0, \quad x \in \partial \Omega, t \in(0, T), \\
u^{\varepsilon}(x, 0)=0, \quad x \in \Omega,
\end{gathered}
$$

где $\Omega_{T}=\Omega \times(0, T), f^{\varepsilon}(x) \in L^{2}(\Omega), \partial / \partial \nu_{A^{\varepsilon}}-$ производная по конормали к $\partial \Omega, \mathrm{a}$ функция $\Phi^{\varepsilon}(x)$ имеет вид

$$
\Phi^{\varepsilon}(x)=\Phi_{m} \cdot \mathbb{I}_{m}^{\varepsilon}(x)+\Phi_{f} \cdot \mathbb{I}_{f}^{\varepsilon}(x) .
$$

В (1.7) $\Phi_{m}, \Phi_{f}$ - строго положительные постоянные, а $\mathbb{I}_{m}^{\varepsilon}(x), \mathbb{I}_{f}^{\varepsilon}(x)$ - характеристические функции областей $\mathscr{M}^{(\varepsilon)}$ и $\mathscr{F}^{(\varepsilon)}$ соответственно. Предполагается, что тензор проводимости $A^{\varepsilon}(x)=\left\{a_{i j}^{\varepsilon}(x)\right\}$ симметричен, $a_{i j}^{\varepsilon}=a_{j i}^{\varepsilon}$, и удовлетворяет условиям

причем

$$
\begin{aligned}
& 0<C_{1}|\xi|^{2} \leqslant\left(A^{\varepsilon}(x) \xi, \xi\right) \leqslant C_{2}|\xi|^{2} \quad \forall \xi \in \mathbb{R}^{n} \backslash\{0\}, \quad x \in \mathscr{F}^{(\varepsilon)}, \\
& 0<c_{1}^{\varepsilon}|\xi|^{2} \leqslant\left(A^{\varepsilon}(x) \xi, \xi\right) \leqslant c_{2}^{\varepsilon}|\xi|^{2} \quad \forall \xi \in \mathbb{R}^{n} \backslash\{0\}, \quad x \in \mathscr{M}^{(\varepsilon)} \text {, }
\end{aligned}
$$

$$
\mu^{\varepsilon} c_{2}^{\varepsilon} \rightarrow 0, \quad \varepsilon \rightarrow 0 .
$$

ЗАМЕчАнИЕ 1.1. В условии (1.8) порядок малости постоянных $c_{1}^{\varepsilon}, c_{2}^{\varepsilon}$, вообще говоря, различен. Так величина $c_{2}^{\varepsilon} / c_{1}^{\varepsilon}$ может быть ограниченной, как в рассматриваемом ниже модельном случае (см. $\S 7$ ), так и неограниченной.

Известно (см., например, [17]), что для любого $\varepsilon>0$ существует единственное решение $u^{\varepsilon}(x, t)$ задачи (1.4)-(1.6), принадлежашее пространству $C\left([0, T] ; H^{1}(\Omega)\right)$. Изучается асимптотическое поведение решений $u^{\varepsilon}(x, t)$ при $\varepsilon \rightarrow 0$. Будет показано, что усреднение задачи (1.4)-(1.6) приводит (как и в случае классической модели двойной пористости) к макроскопической модели с памятью.

Работа построена следующим образом. В $\S 2$ вводится понятие сходимости в областях вырождающейся меры, т.е. удовлетворяющих (1.1). В 33 сформулирован основной результат работы (см. теорему 3.1). Эта теорема доказьватся в $\S \S 4-6$. В $\S 7$ рассматривается случай периодического распределения блоков, для которого коэффициенты усредненной модели получены в явном виде. 


\section{§2. Сходимость в областях вырождающейся меры}

Пусть семейство областей $\mathscr{F}(\varepsilon) \subset \Omega, \varepsilon>0$, удовлетворяет условиям (1.1), (1.2). В этом параграфе, следуя [18] (см. также [19] или [20]), вводится понятие сходимости в областях $\mathscr{F}^{(\varepsilon)}$ при $\varepsilon \rightarrow 0$.

Примем следующие обозначения: $\|\cdot\|_{Q}$ и $\|\cdot\|_{1, Q}$ - нормы в пространствах $L^{2}(Q)$ и $H^{1}(Q)$ соответствнно; $\operatorname{Lip}(M, Q)$ - класс непрерывных в $Q$ функций $u$ таких, что

$$
|u(x)| \leqslant M, \quad|u(x)-u(y)| \leqslant M|x-y|
$$

для любых $x, y \in Q$.

ОПРЕДЕЛЕНИЕ 2.1. Последовательность функций $\left\{u^{\varepsilon} \in L^{2}(\mathscr{F}(\varepsilon))\right\} D_{\mathscr{F}(\varepsilon)}$-сxодится $\kappa$ функии $u \in L^{2}(\Omega)$, если существует аппроксимирующая последовательность $\left\{u_{M} \in \operatorname{Lip}(M, \Omega), M=1,2, \ldots\right\}$, сходящаяся сильно в $L^{2}(\Omega)$ к функции $u$ при $M \rightarrow \infty$, и

$$
\lim _{M \rightarrow \infty} \varlimsup_{\varepsilon \rightarrow 0} \frac{1}{\text { meas } \mathscr{F}(\varepsilon)}\left\|u^{\varepsilon}-u_{M}\right\|_{\mathscr{F}(\varepsilon)}^{2}=0 .
$$

Заметим, что если выполнено условие (1.2), то предельная функция $u$ в определении 2.1 не зависит от выбора аппроксимруюшей последовательности $\left\{u_{M}\right.$, $M=1,2, \ldots\}$ (см. [18]).

Естественньм образом определяется компактность относительно введенной $D_{\mathscr{F}(\varepsilon)}$-схо димости.

ОПРЕДЕЛЕНИЕ 2.2. Последовательность $\left\{u^{\varepsilon} \in L^{2}(\mathscr{F}(\varepsilon))\right\}$ является $D_{\mathscr{F}(\varepsilon)}$-компактным множеством, если из любой ее подпоследовательности можно извлечь

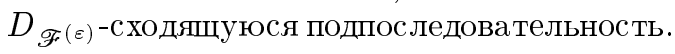

В дальнейшем, мы главным образом будем иметь дело с последовательностями функций $u^{\varepsilon} \in H^{1}(\mathscr{F}(\varepsilon))$, удовлетворяющих условию

$$
\left\|u^{\varepsilon}\right\|_{1, \mathscr{F}(\varepsilon)}^{2} \leqslant C \text { meas } \mathscr{F}^{(\varepsilon)} .
$$

Здесь и далее $C$-постоянная, не зависяшая от $\varepsilon$. Кроме того, в данной работе мы ограничиваемся рассмотрением областей $\mathscr{F}(\varepsilon)$, удовлетворяющих так назьваемому условию "сильной связности" ( $S C$-условию).

ОПРЕДЕЛЕНИЕ 2.3. Будем говорить, что семейство областей $\mathscr{F}^{(\varepsilon)}$ удовлетворяет $S C$-условию, если для любой последовательности $\left\{u^{\varepsilon} \in C^{1}(\mathscr{F}(\varepsilon))\right\}$, удовлетворяющей $(2.2)$, и любого $M, M=1,2, \ldots$, существует семейство подмножеств $G_{M}^{(\varepsilon)} \subset \mathscr{F}^{(\varepsilon)}$ таких, что

$$
u^{\varepsilon} \in \operatorname{Lip}\left(M, \mathscr{F}^{(\varepsilon)} \backslash G_{M}^{(\varepsilon)}\right)
$$

и

$$
\begin{gathered}
\text { meas } G_{M}^{(\varepsilon)}=o\left(\frac{1}{M^{2}}\right) \text { meas } \mathscr{F}^{(\varepsilon)}, \\
\left\|u^{\varepsilon}\right\|_{G_{M}^{(\varepsilon)}}^{2}=o(1) \text { meas } \mathscr{F}^{(\varepsilon)}
\end{gathered}
$$

для достаточно малых $\varepsilon, \varepsilon \leqslant \varepsilon_{0}(M)$, и $M \rightarrow \infty$.

Заметим, что $S C$-условие является обобшением понятия сильной связности, введенного в [21], на области вырождаюшейся меры. Сформулируем теперь до-

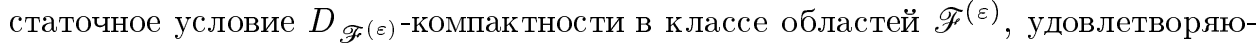
щих (1.2) и $S C$-условию. Справедлива следующая теорема (см. [18]). 
TЕОРема 2.1. Пусть семейство областей $\mathscr{F}(\varepsilon) \subset \Omega$ удовлетворяет $S C$-условию. Тогда любая последовательность $\left\{u^{\varepsilon} \in H^{1}(\mathscr{F}(\varepsilon))\right\}$, удовлетворяющая $(2.2)$, является $D_{\mathscr{F}(\varepsilon)}$-компактным множеством.

\section{§ 3. Формулировка основного результата}

В данном параграфе вводятся локальные энергетические характеристики множеств $\mathscr{F}^{(\varepsilon)}$ и $\mathscr{M}^{(\varepsilon)}$, связанные с начально-краевой задачей (1.4)-(1.6), и формулируется основной результат работы.

Преж де всего, будем предполагать, что

(C.1) существует непрерывная в $\bar{\Omega}$ функиия $\rho(x)>0$ такая, что

$$
\lim _{h \rightarrow 0} \lim _{\varepsilon \rightarrow 0} \mu^{\varepsilon} h^{-n} \operatorname{meas}\left(\mathscr{F}^{(\varepsilon)} \cap K_{h}^{x}\right)=\rho(x)
$$

для любого открытого куба $K_{h}^{x}$ с центром в точке $x \in \Omega$ и ребрами длины $h>0$

(С.2) семейство множеств $\mathscr{F}^{(\varepsilon)}, \varepsilon>0$, удовлетворяет SC-условию (см. определение 2.3 ).

Пусть $K_{h}^{z}$ - открытый куб с центром в точке $z \in \Omega$ и ребрами длины $h>0$, $h \ll 1$. Введем локальную знергетическую характеристику множества $\mathscr{F}(\varepsilon)$ (системы трешин)

$$
E^{\varepsilon, h}(z ; l)=\inf _{v^{\varepsilon}} \mu^{\varepsilon} \int_{K_{h}^{z} \cap \mathscr{F}_{(\varepsilon)}}\left(\left(A^{\varepsilon}(x) \nabla v^{\varepsilon}, \nabla v^{\varepsilon}\right)+h^{-2-\gamma}\left|v^{\varepsilon}-(x-z, l)\right|^{2}\right) d x,
$$

где $\gamma>0, l=\left(l_{1}, l_{2}, \ldots, l_{n}\right) \in \mathbb{R}^{n}$, а инфимуум берется в классе $v^{\varepsilon} \in H^{1}\left(K_{h}^{z} \cap \mathscr{F}^{(\varepsilon)}\right)$. Легко видеть, что функционал $E^{\varepsilon, h}(z ; l)$ квадратичен по $l \in \mathbb{R}^{n}$ и

$$
E^{\varepsilon, h}(z ; l)=\sum_{i, j=1}^{n} a_{i j}^{\varepsilon, h}(z) l_{i} l_{j}
$$

где

$$
\begin{aligned}
a_{i j}^{\varepsilon, h}(z)=\mu^{\varepsilon} \int_{K_{h}^{z} \cap \mathscr{F}(\varepsilon)} & \left(\left(A^{\varepsilon}(x) \nabla v_{i h}^{z \varepsilon}, \nabla v_{j h}^{z \varepsilon}\right)\right. \\
+ & \left.h^{-2-\gamma}\left(\left(v_{i h}^{z \varepsilon}-\left(x_{i}-z_{i}\right)\right)\left(v_{j h}^{z \varepsilon}-\left(x_{j}-z_{j}\right)\right)\right)\right) d x .
\end{aligned}
$$

Здесь $v_{k h}^{z \varepsilon}$ - минимизант функционала в (3.1) при $l=e_{k}$, где $e_{k}$ - единичный вектор оси $x_{k}$. Введем, далее, локальную характеристику

$$
\begin{aligned}
b_{\lambda}^{\varepsilon, h}(z)=\inf _{w^{\varepsilon}} \mu^{\varepsilon} \int_{K_{h}^{z}}( & \left(A^{\varepsilon}(x) \nabla w^{\varepsilon}, \nabla w^{\varepsilon}\right) \\
& \left.+\lambda \Phi_{m} \mathbb{I}_{m}^{\varepsilon}\left|w^{\varepsilon}\right|^{2}+h^{-2-\gamma} \mathbb{I}_{f}^{\varepsilon}\left|w^{\varepsilon}-1\right|^{2}\right) d x
\end{aligned}
$$

где $\mathbb{I}_{m}^{\varepsilon}=\mathbb{I}_{m}^{\varepsilon}(x), \mathbb{I}_{f}^{\varepsilon}=\mathbb{I}_{f}^{\varepsilon}(x)$ - характеристические функции множеств $\mathscr{M}^{(\varepsilon)}$ и $\mathscr{F}^{(\varepsilon)}$, а инфимум берется в классе функций $w^{\varepsilon} \in H^{1}\left(K_{h}^{z}\right)$. В рамках рассматриваемой модели эта характеристика описывает локальные свойства системы, связанные с процессом обмена меж ду трещинами $\left(\mathscr{F}^{(\varepsilon)}\right)$ и матрицей $\left(\mathscr{M}^{(\varepsilon)}\right)$.

Сформулируем теперь основной результат работы. 
ТЕОРема 3.1. Пусть выполнены условия (С.1), (С.2), а также нижеследующие условия.

(С.3) Для некоторого $\gamma=\gamma_{0}, 0<\gamma_{0}<2$, и всех $x \in \Omega$ существуют предель

$$
\lim _{h \rightarrow 0} \frac{\lim }{\varepsilon \rightarrow 0} h^{-n} a_{i j}^{\varepsilon, h}(x)=\lim _{h \rightarrow 0} \varlimsup_{\varepsilon \rightarrow 0} h^{-n} a_{i j}^{\varepsilon, h}(x)=a_{i j}^{*}(x),
$$

әде $a_{i j}^{*} \in C(\bar{\Omega})$. Кроме того, тензор $A^{*}(x)=\left\{a_{i j}^{*}(x)\right\}$ (усредненный тензор проводимости) положительно определен с постоянной коэриитивности $a_{0}>0$, не зависящей от $x \in \Omega$.

(С.4) Для некоторого $\gamma=\gamma_{1}, 0<\gamma_{1}<2$, и любого $\lambda>0$ существует функция $b(x, \lambda), b(\cdot, \lambda) \in C(\bar{\Omega})$, такая, что

$$
\lim _{h \rightarrow 0} \lim _{\varepsilon \rightarrow 0} h^{-n} b_{\lambda}^{\varepsilon, h}(x)=\lim _{h \rightarrow 0} \varlimsup_{\varepsilon \rightarrow 0} h^{-n} b_{\lambda}^{\varepsilon, h}(x)=b(x, \lambda)
$$

$u b(x, \lambda)=O\left(\lambda^{\delta}\right), \delta<1$, npu $\lambda \rightarrow+\infty$.

(С.5) Функиия $f^{\varepsilon}(x)$ равна нулю на $\mathscr{M}^{(\varepsilon)}$ и $D_{\mathscr{F}(\varepsilon)}$-сходится (в смысле определения 2.1) $к$ некоторой функиии $f \in L^{2}(\Omega)$.

Тогда для любого $t \in(0, T)$ решение $u^{\varepsilon}(x, t)$ задачи $(1.4)-(1.6) D_{\mathscr{F}(\varepsilon)}$-сходится к решению и $(x, t)$ следующей начально-краевой задачи:

$$
\begin{gathered}
\rho(x) \Phi_{f} \frac{\partial u}{\partial t}-\operatorname{div}\left(A^{*}(x) \nabla u\right)+\frac{\partial}{\partial t} \int_{0}^{t} B(x, t-\tau) u(x, \tau) d \tau=\rho(x) f(x), \quad x \in \Omega_{T} \\
\frac{\partial u}{\partial \nu_{A^{*}}}(x, t)=0, \quad x \in \partial \Omega, \quad t \in(0, T) \\
u(x, 0)=0, \quad x \in \Omega
\end{gathered}
$$

$2 \partial e$

$$
B(x, t)=\frac{1}{2 \pi i} \lim _{R \rightarrow \infty} \int_{\sigma-i R}^{\sigma+i R} e^{\lambda t} \frac{\widetilde{b}(x, \lambda)}{\lambda} d \lambda, \quad \sigma>0,
$$

а $\widetilde{b}(x, \lambda)$ - аналитическая по $\lambda \in \mathbb{C} \backslash\{\arg \lambda=\pi\}$ функция, совпадающ,ая с $b(x, \lambda)$ для вещественных $\lambda>0$ и $x \in \Omega$.

ЗАмЕчАнИЕ 3.1. В $\S 6$ будет показано, что функция $b(x, \lambda)$, определяемая условием (С.4) для $\lambda>0$, действительно имеет аналитическое продолжение $\tilde{b}(x, \lambda)$ по $\lambda$ в комплексную плоскость с разрезом по отрицательной полуоси, $\mathbb{C} \backslash\{\arg \lambda=\pi\}$, и $\widetilde{b}(x, \lambda)=O\left(|\lambda|^{\delta}\right), \delta<1$, при $|\lambda| \rightarrow \infty$ и $|\arg \lambda-\pi| \geqslant \theta_{0}>0$. Это означает, что предел в (3.7) сушествует, не зависит от $\sigma>0$ и определяет локально суммируемую по $t$ функцию на $[0,+\infty)$.

ЗАмЕчАниЕ 3.2. В теореме 3.1 предполагается, что условия (С.3), (C.4) выполнены для некоторых $\gamma_{0}, \gamma_{1} \in(0,2)$. При этом условии доказано, в частности, неравенство (4.28). Однако, используя идеи работы [21], можно показать, что если (С.3), (С.4) выполняются для некоторых $\gamma_{0}, \gamma_{1} \in(0,2)$, то они выполнены при любых $\gamma>0$.

Теорема 3.1 доказьвается сведением задачи (1.4)-(1.6) с помощью преобразования Лапласа к стационарной краевой задаче для уравнения

$$
-\operatorname{div}\left(A^{\varepsilon}(x) \nabla u^{\varepsilon}\right)+\lambda \Phi^{\varepsilon}(x) u^{\varepsilon}=\lambda^{-1} f^{\varepsilon} .
$$

Стационарная версия теоремы 3.1 при $\lambda>0$ (теорема 5.1) доказана в $\S 5$ вариационньпи методами с использованием предварительных результатов, установленных в $\S 4$. Доказательство теоремы 3.1 завершается в $\S 6$. 
ЗАмечаниЕ 3.3. Для простоты теорема 5.1 будет доказана в предположении, что коэффициенты $a_{i j}^{\varepsilon}\left(A^{\varepsilon}=\left\{a_{i j}^{\varepsilon}\right\}\right)$ и правая часть $f^{\varepsilon}$ в уравнении (1.4) являются достаточно гладкими функциями на множестве $\mathscr{F}^{(\varepsilon)}$, например, $a_{i j}^{\varepsilon}, f^{\varepsilon} \in C^{2}\left(\mathscr{F}^{(\varepsilon)}\right)$. В этом случае решение $u^{\varepsilon}$ стационарной задачи (5.1) (см. §5) и минимизант $v^{\varepsilon}$ функционала в $(3.1)$ принадлежат классам $C^{1}\left(\mathscr{F}^{(\varepsilon)}\right)$ и $C^{1}\left(\mathscr{F}(\varepsilon) \cap K_{h}^{z}\right)$ соответственно. Этот факт будет использован в доказательстве леммы 4.2 и в $\S 5$, там где применяется лемма 4.4. В общем случае в соответствующих местах следует перейти к гладким аппроксимациям этих функций.

\section{§4. Предварительные результаты}

В этом параграфе доказываются вспомогательные результаты, которые будут затем использованы при доказательстве теоремы 3.1 и ее стационарной версии (теоремы 5.1, см. $\S 5$ ).

Покроем область $\Omega$ кубами $K_{h}^{\alpha}=\left\{x:\left|x_{j}-x_{j}^{\alpha}\right|<h / 2\right\}$ с ребрами длины $h>0$, ориентированными вдоль осей координат и с центрами в точках $x^{\alpha}$. Центры $x^{\alpha}$ образуют периодическую решетку с периодом $h^{\prime}=h-h^{1+\gamma / 2}$. Свяжем с этим покрытием разбиение единицы $\left\{\varphi_{h}^{\alpha}(x)\right\}$, которое удовлетворяет следуюшим условиям: $0 \leqslant \varphi_{h}^{\alpha}(x) \leqslant 1 ; \varphi_{h}^{\alpha}(x)=0$ при $x \notin K_{h}^{\alpha} ; \varphi_{h}^{\alpha}(x)=1$ при $x \in K_{h}^{\alpha} \backslash$ $\bigcup_{\alpha \neq \beta} K_{h}^{\beta} ; \sum_{\alpha} \varphi_{h}^{\alpha}(x)=1$ при $x \in \Omega$ и $\left|\nabla \varphi_{h}^{\alpha}\right| \leqslant C h^{-1-\gamma / 2}$.

ЛЕмма 4.1. Пусть выполнены условия (С.1), (С.4) теоремы 3.1. Тогда для любого $h>0$ существуют множества $G_{h}^{(\varepsilon)} \subset \mathscr{F}(\varepsilon)$ и функции $Y_{h}^{\varepsilon} \in H^{1}(\Omega)$ такие, что

(A) $0 \leqslant Y_{h}^{\varepsilon}(x) \leqslant 1, x \in \Omega$;

(B) $Y_{h}^{\varepsilon}(x)=1, x \in \mathscr{F}^{(\varepsilon)} \backslash G_{h}^{(\varepsilon)}$;

(C) $\varlimsup_{\varepsilon \rightarrow 0} \mu^{\varepsilon}$ meas $G_{h}^{(\varepsilon)}=O\left(h^{\gamma}\right)$ npu $h \rightarrow 0$;

(D) для любой функиии $\eta \in C(\bar{\Omega})$

$$
\begin{aligned}
& \varlimsup_{\varepsilon \rightarrow 0} \mu^{\varepsilon} \int_{\Omega} \eta^{2}\left(\left(A^{\varepsilon} \nabla Y_{h}^{\varepsilon}, \nabla Y_{h}^{\varepsilon}\right)+\lambda \Phi^{\varepsilon}(x)\left|Y_{h}^{\varepsilon}\right|^{2}\right) d x \\
& \leqslant \int_{\Omega}\left(b(x, \lambda)+\lambda \rho(x) \Phi_{f}\right) \eta^{2} d x+o(1) \quad n p u \quad h \rightarrow 0 .
\end{aligned}
$$

ДоКАЗАТЕЛЬСТВО. Пусть функция $w_{h}^{\varepsilon \alpha}$ минимизирует функционал в (3.3) при $z=x^{\alpha}$. Из условия (С.4) следует, что при $h \rightarrow 0$

$$
\begin{gathered}
\varlimsup_{\varepsilon \rightarrow \infty} \mu^{\varepsilon} \int_{K_{h}^{\alpha} \backslash K_{h^{\prime}}^{\alpha}}\left(\left(A^{\varepsilon}(x) \nabla w_{h}^{\varepsilon \alpha}, \nabla w_{h}^{\varepsilon \alpha}\right)+\lambda \mathbb{I}_{m}^{\varepsilon} \Phi_{m}\left|w_{h}^{\varepsilon \alpha}\right|^{2}\right) d x=o\left(h^{n}\right), \\
\varlimsup_{\varepsilon \rightarrow \infty} \mu^{\varepsilon} \int_{K_{h}^{\alpha} \backslash K_{h^{\prime}}^{\alpha}} \mathbb{I}_{f}^{\varepsilon}\left|w_{h}^{\varepsilon \alpha}-1\right|^{2} d x=o\left(h^{n+2+\gamma}\right) .
\end{gathered}
$$

В дополнение к этому, пользуясь условиями (C.1) и (C.4), получаем

$$
\begin{gathered}
\varlimsup_{\varepsilon \rightarrow \infty} \mu^{\varepsilon} \int_{K_{h}^{\alpha}}\left(\left(A^{\varepsilon}(x) \nabla w_{h}^{\varepsilon \alpha}, \nabla w_{h}^{\varepsilon \alpha}\right)+\lambda \Phi^{\varepsilon}(x)\left|w_{h}^{\varepsilon \alpha}\right|^{2}\right) d x \\
\leqslant h^{n}\left(b\left(x^{\alpha}, \lambda\right)+\lambda \rho\left(x^{\alpha}\right)\right)+o\left(h^{n}\right) \text { при } h \rightarrow 0 .
\end{gathered}
$$


Кроме того, так как $w_{h}^{\varepsilon \alpha}$ минимизирует функционал в $(3.3)$, то $0 \leqslant w_{h}^{\varepsilon \alpha}(x) \leqslant 1$ и

$$
\varlimsup_{\varepsilon \rightarrow \infty} \mu^{\varepsilon} \text { meas } \widehat{B}_{h}^{\varepsilon \alpha} \leqslant C h^{n+\gamma},
$$

где $\widehat{B}_{h}^{\varepsilon \alpha}=\left\{x \in K_{h}^{\alpha} \cap \mathscr{F}^{\varepsilon}: w_{h}^{\varepsilon \alpha}(x) \leqslant 1-h\right\}$.

Рассмотрим теперь функции

$$
\widehat{w}_{h}^{\varepsilon \alpha}(x)=\frac{1}{1-h} \times \begin{cases}1-h, & w_{h}^{\varepsilon \alpha}(x) \geqslant 1-h ; \\ w_{h}^{\varepsilon \alpha}(x) & \text { в противном случае. }\end{cases}
$$

Легко видеть, что $\left|\widehat{w}_{h}^{\varepsilon \alpha}-1\right| \leqslant\left|w_{h}^{\varepsilon \alpha}-1\right|$ и функции $\widehat{w}_{h}^{\varepsilon \alpha}$ удовлетворяют оценкам (4.1)-(4.3). Положим

$$
G_{h}^{(\varepsilon)}=\bigcup_{\alpha} \widehat{B}_{h}^{\varepsilon \alpha}, \quad Y_{h}^{\varepsilon}(x)=\sum_{\alpha} \widehat{w}_{h}^{\varepsilon \alpha}(x) \varphi_{h}^{\alpha}(x) .
$$

Тогда, используя свойства функций $\widehat{w}_{h}^{\varepsilon \alpha}$ и разбиения единицы $\left\{\varphi_{h}^{\alpha}\right\}$ и принимая во внимание оценку (4.4), нетрудно показать, что функции $Y_{h}^{\varepsilon}(x)$ и множества $G_{h}^{(\varepsilon)}$ удовлетворяют условиям (A)-(D) леммы 4.1. Лемма 4.1 доказана.

ЛЕмма 4.2. Пусть выполнены условия (С.2), (С.3) теоремь 3.1, и пусть $\eta$ - гладкая в $\bar{\Omega}$ функиия. Тогда для любого $h>0$ и $M \in\{1,2, \ldots\}$ существуют функции $W_{M h}^{\varepsilon} \in H^{1}(\Omega)$ такие, что

(A) $\left|W_{M h}^{\varepsilon}(x)-\eta(x)\right| \leqslant C M h, x \in \Omega$;

(B) $\left|W_{M h}^{\varepsilon}(x)-W_{M h}^{\varepsilon}(y)\right| \leqslant C M|x-y|, x, y \in \Omega$;

(C) $\varlimsup_{M \rightarrow \infty} \varlimsup_{h \rightarrow 0} \varlimsup_{\varepsilon \rightarrow 0} \mu^{\varepsilon} \int_{\mathscr{F}(\varepsilon)}\left(A^{\varepsilon}(x) \nabla W_{M h}^{\varepsilon}, \nabla W_{M h}^{\varepsilon}\right) d x \leqslant \int_{\Omega}\left(A^{*}(x) \nabla \eta, \nabla \eta\right) d x$.

ДокАЗАТЕЛЬСТво. Пусть $v_{k h}^{\alpha \varepsilon}$ - функция, минимизируюшая функционал в (3.1) при $l=e_{k}$, где $e_{k}-$ орт оси $x_{k}$. Будем предполагать, что $v_{k h}^{\alpha \varepsilon}(x)=x_{k}-x_{k}^{\alpha}$, если $K_{h}^{\alpha} \cap\left(\mathbb{R}^{n} \backslash \Omega\right) \neq \varnothing$. Поскольку $v_{k h}^{\alpha \varepsilon}$ минимизирует функционал в (3.1),

$$
\sup _{x \in K_{h}^{\alpha} \cap \mathscr{F}(\varepsilon)}\left|v_{k h}^{\alpha \varepsilon}(x)\right| \leqslant \frac{h}{2} .
$$

Кроме того, в силу условия (С.3) для достаточно малых $\varepsilon, \varepsilon \leqslant \widehat{\varepsilon}(h)$, имеем

$$
\begin{gathered}
\mu^{\varepsilon} \int_{\left(K_{h}^{\alpha} \backslash K_{h^{\prime}}^{\alpha}\right) \cap \mathscr{F}(\varepsilon)}\left|\nabla v_{k h}^{\alpha \varepsilon}\right|^{2} d x=o\left(h^{n}\right), \\
\mu^{\varepsilon} \int_{\left(K_{h}^{\alpha} \backslash K_{h^{\prime}}^{\alpha}\right) \cap \mathscr{F}_{(\varepsilon)}}\left|v_{k h}^{\alpha \varepsilon}-\left(x_{k}-x_{k}^{\alpha}\right)\right|^{2} d x=o\left(h^{n+2+\gamma}\right), \\
\mu^{\varepsilon} \int_{K_{h}^{\alpha} \cap \mathscr{F}(\varepsilon)}\left(A^{\varepsilon}(x) \nabla v_{i h}^{\alpha \varepsilon}, \nabla v_{j h}^{\alpha \varepsilon}\right) d x \leqslant h^{n} a_{i j}\left(x^{\alpha}\right)+o\left(h^{n}\right)
\end{gathered}
$$

при $h \rightarrow 0$. Оценки (4.7)-(4.9) равномерны по $x^{\alpha}$ на любом компактном подмножестве $\Omega$.

Рассмотрим функции

$$
w_{h}^{\varepsilon}(x)=\sum_{\alpha}\left(\eta\left(x^{\alpha}\right)+\sum_{k=1}^{n} \frac{\partial \eta}{\partial x_{k}}\left(x^{\alpha}\right) v_{k h}^{\alpha \varepsilon}(x)\right) \varphi_{h}^{\alpha}(x) .
$$


Заметим, что правую часть (4.10) можно записать в виде

$$
\begin{aligned}
w_{h}^{\varepsilon}(x)= & \eta(x)+\sum_{\alpha}\left(\eta\left(x^{\alpha}\right)+\sum_{k=1}^{n} \frac{\partial \eta}{\partial x_{k}}\left(x^{\alpha}\right)\left(x-x_{k}\right)-\eta(x)\right. \\
& \left.+\sum_{k=1}^{n} \frac{\partial \eta}{\partial x_{k}}\left(x^{\alpha}\right)\left(v_{k h}^{\alpha \varepsilon}(x)-\left(x-x_{k}\right)\right)\right) \varphi_{h}^{\alpha}(x) .
\end{aligned}
$$

Тогда, пользуясь (4.7)-(4.9) и свойствами функций $\varphi_{h}^{\alpha}$, получаем

$$
\mu^{\varepsilon} \int_{\mathscr{F}(\varepsilon)}\left(A^{\varepsilon}(x) \nabla w_{h}^{\varepsilon}, \nabla w_{h}^{\varepsilon}\right) d x \leqslant \int_{\Omega}\left(A^{*}(x) \nabla \eta, \nabla \eta\right) d x+o(1)
$$

при $\varepsilon \leqslant \widehat{\varepsilon}(h)$ и $h \rightarrow 0$. Кроме того, согласно (4.6) и (4.10) имеем

$$
\sup _{x \in \mathscr{F}(\varepsilon)}\left|w_{h}^{\varepsilon}(x)-\eta(x)\right| \leqslant C h .
$$

Поскольку области $\mathscr{F}^{(\varepsilon)}$ удовлетворяют $S C$-условию (см. определение 2.3 ), для любого $M=1,2, \ldots$ сушествуют множества $Q_{M h}^{(\varepsilon)}$ и функции $W_{M h}^{\varepsilon} \in \operatorname{Lip}(M, \Omega)$ такие, что $W_{M h}^{\varepsilon}=w_{h}^{\varepsilon}$ в $\mathscr{F}^{(\varepsilon)} \backslash Q_{M h}^{(\varepsilon)}$ и

$$
\varlimsup_{M \rightarrow \infty} M^{2} \varlimsup_{h \rightarrow 0} \varlimsup_{\varepsilon \rightarrow 0} \mu^{\varepsilon} \text { meas } Q_{M h}^{(\varepsilon)}=0 .
$$

Предположим, что

$$
\left(\mathscr{F}^{(\varepsilon)} \backslash Q_{M h}^{(\varepsilon)}\right) \cap K_{h}^{\alpha} \neq \varnothing
$$

выполнено для любого $\alpha$. Тогда утверждения леммы следуют из (4.11), (4.12) и свойств функций $W_{M h}^{\varepsilon}$.

Предположим теперь, что условие (4.14) не вьполнено для $\alpha$ из некоторого множества $\Upsilon$. Пусть $\left\{\phi_{h}^{\alpha}\right\}$ - разбиение единицы такое, что $0 \leqslant \phi_{h}^{\alpha} \leqslant 1, \phi_{h}^{\alpha}(x)=0$ при $x \notin\left\{x:\left|x_{j}-x_{j}^{\alpha}\right| \geqslant h\right\}, \sum_{\alpha} \phi_{h}^{\alpha}=1,\left|\nabla \phi_{h}^{\alpha}\right| \leqslant C h^{-1}$. Переопределим функции $W_{M h}^{\varepsilon}$ и множества $Q_{M h}^{(\varepsilon)}$, введенные вьше, следуюшим образом:

$$
\begin{gathered}
W_{M h}^{\varepsilon}(x):=W_{M h}^{\varepsilon}(x)+\left(\eta(x)-W_{M h}^{\varepsilon}(x)\right) \sum_{\alpha \in \Upsilon} \phi_{h}^{\alpha}(x), \\
Q_{M h}^{(\varepsilon)}:=Q_{M h}^{(\varepsilon)} \cup\left(\mathscr{F}(\varepsilon) \cap \bigcup_{\alpha \in \Upsilon \cup \Upsilon^{\prime}} K_{h}^{\alpha}\right),
\end{gathered}
$$

где $\Upsilon^{\prime}$ - множество $\alpha^{\prime}$ таких, что $K_{h}^{\alpha^{\prime}}$ является ближайшим соседним с кубом $K_{h}^{\alpha}$ для некоторого $\alpha \in \Upsilon$. Заметим, что $\left|\nabla W_{M h}^{\varepsilon}\right| \leqslant C M$ для достаточно малых $\varepsilon$ и $h$, а множества $Q_{M h}^{(\varepsilon)}$ удовлетворяют (4.13). Таким образом, утверж дения леммы вьполнены и в этом случае (по тем же соображениям, что и вьше). Лемма 4.2 доказана.

ЛЕмма 4.3. Пусть выполнены условия (С.1) и (С.4) теоремы 3.1. Тогда для любой функиии $w \in H^{1}(\Omega)$ существует последовательность $\left\{w^{\varepsilon} \in C^{1}(\Omega)\right\}$, которая $D_{\mathscr{F}(\varepsilon)}$-сходится $\kappa w$, причем

$$
\varlimsup_{\varepsilon \rightarrow 0} I^{(\varepsilon)}\left[w^{\varepsilon}\right] \equiv \varlimsup_{\varepsilon \rightarrow 0} \mu^{\varepsilon} \int_{\Omega}\left(\left(A^{\varepsilon}(x) \nabla w^{\varepsilon}, \nabla w^{\varepsilon}\right)+\lambda \Phi^{\varepsilon}(x)\left|w^{\varepsilon}\right|^{2}\right) d x \leqslant \nu\|w\|_{1, \Omega}^{2},
$$

әде $\nu>0$ не зависит от $w$. 
ДокАЗАТЕЛЬСтво. Предположим вначале, что $w \in C^{\infty}(\Omega)$. Тогда последовательность $\left\{w^{\varepsilon}\right\}$ строится следуюшим образом. Рассмотрим функцию

$$
w_{h}^{\varepsilon}(x)=w(x) Y_{h}^{\varepsilon}(x),
$$

где функция $Y_{h}^{\varepsilon}(x)$ определена в лемме 4.1. Согласно лемме 4.1 сушествуют множества $G_{h}^{(\varepsilon)}\left(\subset \mathscr{F}^{(\varepsilon)}\right)$ такие, что meas $G_{h}^{(\varepsilon)} \leqslant C h^{\gamma}$ meas $\mathscr{F}^{(\varepsilon)}(\varepsilon \leqslant \widetilde{\varepsilon}(h)), w_{h}^{\varepsilon}(x)=$ $w(x)$ при $x \in \mathscr{F}^{(\varepsilon)} \backslash G_{h}^{(\varepsilon)}$ и

$$
\begin{gathered}
\int_{G_{h}^{(\varepsilon)}}\left|w_{h}^{\varepsilon}-w\right|^{2} d x \leqslant \int_{G_{h}^{(\varepsilon)}}|w|^{2} d x, \\
I^{(\varepsilon)}\left[w_{h}^{\varepsilon}\right] \leqslant \mu^{\varepsilon} \int_{\mathscr{F}(\varepsilon) \backslash G_{h}^{(\varepsilon)}}|\nabla w|^{2} d x+\int_{\Omega}(b(x, \lambda)+\lambda \rho(x))|w|^{2} d x+\xi(h), \quad \varepsilon \leqslant \widetilde{\varepsilon}(h),
\end{gathered}
$$

где $\xi(h) \rightarrow 0$ при $h \rightarrow 0$. Причем в силу условий $($ C.1) и (1.2)

$\mu^{\varepsilon} \int_{\mathscr{F}(\varepsilon)}|\nabla w|^{2} d x \rightarrow \int_{\Omega} \rho(x)|\nabla w|^{2} d x, \mu^{\varepsilon} \int_{\mathscr{F}(\varepsilon)}|w|^{2} d x \rightarrow \int_{\Omega} \rho(x)|w|^{2} d x, \quad \varepsilon \rightarrow 0$.

Ясно, что найдется последовательность $\left\{\widehat{\varepsilon}_{j}\right\}, \widehat{\varepsilon}_{j} \downarrow 0$, такая, что $\widehat{\varepsilon}_{j} \leqslant \widetilde{\varepsilon}(1 / j)$. Положим

$$
w^{\varepsilon}=\left.w_{h}^{\varepsilon}\right|_{h=1 / j} \text { при } \widehat{\varepsilon}_{j} \geqslant \varepsilon>\widehat{\varepsilon}_{j+1} .
$$

Из (4.16), (4.17) следует, что последовательность $\left\{w^{\varepsilon}\right\}$ удовлетворяет (4.15), где $\nu=C \sup _{x \in \Omega}(b(x, \lambda)+\rho(x))$ и

$$
\lim _{\varepsilon \rightarrow 0} \frac{1}{\operatorname{meas} \mathscr{F}(\varepsilon)} \int_{\mathscr{F}(\varepsilon)}\left|w^{\varepsilon}-w\right|^{2} d x=0
$$

Очевидно, что функции $w^{\varepsilon}$ могут быть аппроксимированы гладкими функциями так, чтобы (4.15) и последнее соотношение оставались справедливыми.

Пусть теперь $w$ - произвольная функция из $H^{1}(\Omega)$. Аппроксимируем $w$ гладкими функциями $w_{M} \in \operatorname{Lip}(M, \Omega), M=1,2, \ldots$, таким образом, что

$$
\left\|w-w_{M}\right\|_{1, \Omega} \leqslant \frac{1}{k(M)}, \quad k(M) \rightarrow 0, \quad M \rightarrow \infty .
$$

Согласно предыдушему для любого фиксированного $M \in\{1,2, \ldots\}$ найдется последовательность $\left\{w_{M}^{\varepsilon}\right\}$, удовлетворяющая следующим требованиям:

$$
\begin{gathered}
\frac{1}{\operatorname{meas} \mathscr{F}(\varepsilon)} \int_{\mathscr{F}(\varepsilon)}\left|w_{M}^{\varepsilon}-w_{M}\right|^{2} d x \leqslant \frac{1}{M}, \\
I^{(\varepsilon)}\left[w_{M}^{\varepsilon}\right] \leqslant \nu\left(1+\frac{1}{k(M)}\right)\|w\|_{1, \Omega}^{2}
\end{gathered}
$$

при $\varepsilon \leqslant \delta(M)$, где $\delta(M) \rightarrow 0$ при $M \rightarrow \infty$. Причем вследствие (1.2) $\delta(M)$ может быть выбрано таким образом, чтобы при $\varepsilon \leqslant \delta(M)$

$$
\frac{1}{\operatorname{meas} \mathscr{F}(\varepsilon)} \int_{\mathscr{F}(\varepsilon)}\left|w_{M}-w_{S}\right|^{2} d x \leqslant C\left\|w_{M}-w_{S}\right\|_{\Omega}^{2}, \quad \forall S=1, \ldots, M-1,
$$


где постоянная $C$ не зависит от $M$. Выберем последовательность $\left\{\widehat{\delta}_{j}\right\}, \widehat{\delta}_{j} \downarrow 0$, такую, что $\widehat{\delta}_{j} \leqslant \delta(j)$, и определим

$$
w^{\varepsilon}=w_{M}^{\varepsilon} \quad \text { при } \quad \varepsilon \in\left(\widehat{\delta}_{M+1}, \widehat{\delta}_{M}\right]
$$

Легко видеть, что $\left\{w^{\varepsilon}\right\}$ удовлетворяет (4.15). Кроме того,

$$
\begin{aligned}
& \frac{1}{\operatorname{meas} \mathscr{F}(\varepsilon)} \int_{\mathscr{F}(\varepsilon)}\left|w^{\varepsilon}-w_{M_{0}}\right|^{2} d x \\
& \quad \leqslant \frac{2}{\operatorname{meas} \mathscr{F}(\varepsilon)} \int_{\mathscr{F}(\varepsilon)}\left|w_{M}^{\varepsilon}-w_{M}\right|^{2} d x+2 C\left\|w_{M}-w_{M_{0}}\right\|_{\Omega}^{2}
\end{aligned}
$$

для любых $M_{0}$ и любого $\varepsilon \in\left(\widehat{\delta}_{M+1}, \widehat{\delta}_{M}\right]$. Здесь $C$ - постоянная, фигурируюшая в (4.20). Следовательно, согласно (4.18)-(4.20) последовательность $\left\{w^{\varepsilon}\right\}$ $D_{\mathscr{F}(\varepsilon)}$-сходится к $w$. Лемма 4.3 доказана.

Лемма 4.4. Пусть выполнень условия (С.1)-(С.3) теоремы 3.1, и пусть последовательность $\left\{v^{\varepsilon} \in C^{1}\left(\mathscr{F}^{(\varepsilon)}\right)\right\}$ удовлетворяет $(2.2)$. Тогда существует семейство непрерывных в $\Omega$ функиий $\left\{v_{M}^{\varepsilon}\right\}, M=1,2, \ldots$, удовлетворяюших следуюшим условиям:

(A) $\lim _{M \rightarrow \infty} M^{2} \varlimsup_{\varepsilon \rightarrow 0} \mu^{\varepsilon} \operatorname{meas}\left\{x \in \mathscr{F}^{(\varepsilon)}: v^{\varepsilon}(x) \neq v_{M}^{\varepsilon}(x)\right\}=0$

(B) существует постоянная $C>0$, которая не зависит от $\varepsilon$ и $M$, такая, что $v_{M}^{\varepsilon} \in \operatorname{Lip}(C M, \Omega)$;

(C) для любого $M$ существует подпоследовательность $\left\{v_{M}^{\varepsilon_{j}}\right\}, \varepsilon_{j} \rightarrow 0$, равномерно сходящаяся в $\Omega \kappa$ некоторой функиии $v_{M} \in \operatorname{Lip}(C M, \Omega)$;

(D) для любой последовательности множеств $G_{M}^{(\varepsilon)} \subset \mathscr{F}(\varepsilon)$ таких, что

$$
\lim _{M \rightarrow \infty} M^{2} \varlimsup_{\varepsilon \rightarrow 0} \mu^{\varepsilon} \text { meas } G_{M}^{(\varepsilon)}=0
$$

имеем

$$
\begin{gathered}
\underset{\varepsilon=\lim _{j} \rightarrow 0}{ } \mu^{\varepsilon} \int_{\mathscr{F}(\varepsilon) \backslash G_{M}^{(\varepsilon)}}\left(A^{\varepsilon}(x) \nabla v^{\varepsilon}, \nabla v^{\varepsilon}\right) d x \\
\geqslant \int_{\Omega}\left(A^{*}(x) \nabla v_{M}, \nabla v_{M}\right) d x-\xi(M)
\end{gathered}
$$

әде $\xi(M) \rightarrow 0$ при $M \rightarrow \infty$, причем

$$
\lim _{M \rightarrow \infty} \varlimsup_{\varepsilon \rightarrow 0} \mu^{\varepsilon}\left\|v^{\varepsilon}\right\|_{G_{M}^{(\varepsilon)}}^{2}=0
$$

для любой последовательности множеств $G_{M}^{(\varepsilon)} \subset \mathscr{F}^{(\varepsilon)}$, удовлетворяющ, их (4.21). 
ДокАЗАТЕЛЬСТво. Из условия (С.2) следует, что функции $v^{\varepsilon}$ принадлежат классу $\operatorname{Lip}\left(M, \mathscr{F}(\varepsilon) \backslash Q_{M}^{(\varepsilon)}\right)$ на некотором множестве $\mathscr{F}(\varepsilon) \backslash Q_{M}^{(\varepsilon)}$, причем

$$
\mu^{\varepsilon} \operatorname{meas} Q_{M}^{(\varepsilon)}=o\left(\frac{1}{M^{2}}\right), \quad \mu^{\varepsilon}\left\|v^{\varepsilon}\right\|_{Q_{M}^{(\varepsilon)}}^{2}=o(1)
$$

при $\varepsilon \leqslant \varepsilon_{0}(M), M \rightarrow \infty$. Отсюда следует, что соотношение (4.23) вьполнено. Кроме того, согласно теореме Уитни (см. [22]) функции $v^{\varepsilon}$ имеют продолжения $v_{M}^{\varepsilon} \in \operatorname{Lip}(C M, \Omega)$ такие, что $v^{\varepsilon}(x)=v_{M}^{\varepsilon}(x)$ при $x \in \mathscr{F}^{(\varepsilon)} \backslash Q_{M}^{(\varepsilon)}$, где постоянная $C>0$ не зависит от $\varepsilon$ и $M$. Это означает, что множество $\left\{v_{M}^{\varepsilon}\right\}$ компактно в $C(\bar{\Omega})$ для любого фиксированного $M$; следовательно, найдется подпоследовательность $\left\{v_{M}^{\varepsilon_{j}}\right\}, \varepsilon_{j} \rightarrow 0$, сходящаяся равномерно в $\Omega$ к некоторой функции $v_{M} \in \operatorname{Lip}(C M, \Omega)$. Таким образом, семейство функций $\left\{v_{M}^{\varepsilon}\right\}$ удовлетворяет условиям (А)-(C).

Остается доказать утверждение (D). Пусть $v_{M \delta}$ - гладкая в $\Omega$ функция такая, что

$$
\left\|v_{M \delta}-v_{M}\right\|_{1, \Omega}<\delta, \quad \delta>0
$$

Построим последовательность $\left\{v_{M \delta}^{\varepsilon}\right\}$, удовлетворяюшую соотношениям

$$
\begin{gathered}
\varlimsup_{\varepsilon \rightarrow 0} \mu^{\varepsilon}\left\|v_{M \delta}^{\varepsilon}\right\|_{1, \mathscr{F}(\varepsilon)}^{2} \leqslant C \delta^{2}, \\
\varlimsup_{\varepsilon \rightarrow 0} \mu^{\varepsilon}\left\|v_{M \delta}^{\varepsilon}-\left(v_{M \delta}-v_{M}\right)\right\|_{\mathscr{F}(\varepsilon)}^{2}=0 .
\end{gathered}
$$

Для этого введем гладкие функции $w_{k}$ такие, что

$$
\int_{\Omega}\left|\nabla w_{k}\right|^{2} d x \leqslant \delta^{2}+\frac{1}{k}, \quad \int_{\Omega}\left|w_{k}-\left(v_{M \delta}-v_{M}\right)\right|^{2} d x \leqslant \frac{1}{k}
$$

для любого $k=1,2, \ldots$.

Тогда, поскольку $w_{k},\left|\nabla w_{k}\right|, v_{M \delta}$ и $v_{M}$ непрерывны в $\Omega$, из $(1.2)$ и условия (C.1) получаем

$$
\begin{gathered}
\lim _{\varepsilon \rightarrow 0} \mu^{\varepsilon} \int_{\mathscr{F}(\varepsilon)}\left|\nabla w_{k}\right|^{2} d x=\int_{\Omega}\left|\nabla w_{k}\right|^{2} \rho(x) d x, \\
\lim _{\varepsilon \rightarrow 0} \mu^{\varepsilon} \int_{\mathscr{F}(\varepsilon)}\left|w_{k}-\left(v_{M \delta}-v_{M}\right)\right|^{2} d x=\int_{\Omega}\left|w_{k}-\left(v_{M \delta}-v_{M}\right)\right|^{2} \rho(x) d x .
\end{gathered}
$$

Отсюда следует, что существует последовательность $\left\{\widehat{\varepsilon}_{k}\right\}, \widehat{\varepsilon}_{k} \downarrow 0$, такая, что

$$
\begin{gathered}
\mu^{\varepsilon} \int_{\mathscr{F}(\varepsilon)}\left|\nabla w_{k}\right|^{2} d x \leqslant C\left(\delta^{2}+\frac{1}{k}\right), \\
\mu^{\varepsilon} \int_{\mathscr{F}(\varepsilon)}\left|w_{k}-\left(v_{M \delta}-v_{M}\right)\right|^{2} d x \leqslant C \frac{1}{k}
\end{gathered}
$$

при $\varepsilon<\widehat{\varepsilon}_{k}$. Определим $v_{M \delta}^{\varepsilon}=w_{k}$ при $\widehat{\varepsilon}_{k+1} \leqslant \varepsilon<\widehat{\varepsilon}_{k}$, тогда последовательность $\left\{v_{M \delta}^{\varepsilon}\right\}$ удовлетворяет (4.25), (4.26). Согласно (4.25) имеем

$$
\begin{aligned}
& \lim _{\varepsilon \rightarrow 0} \mu^{\varepsilon} \int_{\mathscr{F}(\varepsilon)}\left(A^{\varepsilon}(x) \nabla v_{M}^{\varepsilon}, \nabla v_{M}^{\varepsilon}\right) d x \\
& \quad \geqslant \frac{\lim _{\varepsilon \rightarrow 0}}{\varepsilon} \int_{\mathscr{F}(\varepsilon)}\left(A^{\varepsilon}(x) \nabla\left(v_{M}^{\varepsilon}+v_{M \delta}^{\varepsilon}\right), \nabla\left(v_{M}^{\varepsilon}+v_{M \delta}^{\varepsilon}\right)\right) d x+\xi_{1}(\delta),
\end{aligned}
$$

где $\xi_{1}(\delta) \rightarrow 0$ при $\delta \rightarrow 0$. 
Покроем область $\Omega$ непересекающимися во внутренних точках кубами $\widetilde{K}_{h}^{\alpha}=$ $\left\{x:\left|x_{l}-\widetilde{x}_{l}^{\alpha}\right| \leqslant h / 2\right\}$. Пусть $\widetilde{K}_{h}^{\alpha} \cap \partial \Omega=\varnothing$ и $\psi_{M \delta}^{\varepsilon}(x)=v_{M}^{\varepsilon}(x)+v_{M \delta}^{\varepsilon}(x)-v_{M \delta}\left(\widetilde{x}^{\alpha}\right)$, тогда имеем

$$
\begin{aligned}
& \int_{\mathscr{F}(\varepsilon) \cap \widetilde{K}_{h}^{\alpha}}\left(A^{\varepsilon} \nabla \psi_{M \delta}^{\varepsilon}, \nabla \psi_{M \delta}^{\varepsilon}\right) d x \\
&=\int_{\mathscr{F}(\varepsilon) \cap \widetilde{K}_{h}^{\alpha}}\left(\left(A^{\varepsilon} \nabla \psi_{M \delta}^{\varepsilon}, \nabla \psi_{M \delta}^{\varepsilon}\right)\right. \\
&\left.+h^{-2-\gamma}\left|\psi_{M \delta}^{\varepsilon}-\left(\nabla v_{M \delta}\left(\widetilde{x}^{\alpha}\right), x-\widetilde{x}^{\alpha}\right)\right|^{2}\right) d x-\xi_{2}^{\alpha}(\varepsilon, h, M), \\
& \varlimsup_{\varepsilon=\varepsilon_{j} \rightarrow 0} \xi_{2}^{\alpha}(\varepsilon, h, M)=O\left(h^{n+2-\gamma}\right), \quad h \rightarrow 0 .
\end{aligned}
$$

Здесь мы воспользовались соотношением (4.26) и тем фактом, что функции $v_{M}^{\varepsilon_{j}}$ равномерно в $\Omega$ сходятся к $v_{M}$ при $\varepsilon_{j} \rightarrow 0$. Следовательно, из условия (С.3) теоремы 3.1 вытекает, что

$$
\begin{gathered}
\underline{\lim }_{\varepsilon=\varepsilon_{j} \rightarrow 0} \mu^{\varepsilon} \int_{\mathscr{F}(\varepsilon)}\left(A^{\varepsilon}(x) \nabla\left(v_{M}^{\varepsilon}+v_{M \delta}^{\varepsilon}\right), \nabla\left(v_{M}^{\varepsilon}+v_{M \delta}^{\varepsilon}\right)\right) d x \\
\geqslant \int_{\Omega}\left(A^{*}(x) \nabla v_{M \delta}, \nabla v_{M \delta}\right) d x
\end{gathered}
$$

Переходя теперь к пределу в (4.28) при $\delta \rightarrow 0$ и используя $(4.24),(4.27)$, получаем

$$
\underline{\lim }_{\varepsilon=\varepsilon_{j} \rightarrow 0} \mu^{\varepsilon} \int_{\mathscr{F}(\varepsilon)}\left(A^{\varepsilon}(x) \nabla v_{M}^{\varepsilon}, \nabla v_{M}^{\varepsilon}\right) d x \geqslant \int_{\Omega}\left(A^{*}(x) \nabla v_{M}, \nabla v_{M}\right) d x .
$$

Наконец, легко видеть, что

$$
\begin{aligned}
& \underbrace{}_{\varepsilon=\varepsilon_{j} \rightarrow 0} \mu^{\varepsilon} \int_{\mathscr{F}(\varepsilon) \backslash G_{M}^{(\varepsilon)}}\left(A^{\varepsilon}(x) \nabla v^{\varepsilon}, \nabla v^{\varepsilon}\right) d x \\
& \quad \geqslant \underline{\lim }_{\varepsilon=\varepsilon_{j} \rightarrow 0} \mu^{\varepsilon} \int_{\mathscr{F}(\varepsilon)}\left(A^{\varepsilon}(x) \nabla v_{M}^{\varepsilon}, \nabla v_{M}^{\varepsilon}\right) d x-\xi(M),
\end{aligned}
$$

где

$$
\xi(M)=\varlimsup_{\varepsilon \rightarrow 0} \mu^{\varepsilon} \int_{G_{M}^{(\varepsilon)} \cup Q_{M}^{(\varepsilon)}}\left(A^{\varepsilon}(x) \nabla v_{M}^{\varepsilon}, \nabla v_{M}^{\varepsilon}\right) d x=o(1), \quad M \rightarrow \infty .
$$

Утверждение (D) леммы 4.4 следует из (4.29) и (4.30). Лемма 4.4 доказана.

\section{§5. Стационарная версия теоремы 3.1}

В этом параграфе мы установим стационарную версию теоремы 3.1. Этот результат является базовьм для доказательства основной теоремы 3.1 .

Рассмотрим краевую задачу

$$
\begin{gathered}
-\operatorname{div}\left(A^{\varepsilon}(x) \nabla u^{\varepsilon}\right)+\lambda \Phi^{\varepsilon}(x) u^{\varepsilon}=\lambda^{-1} f^{\varepsilon}(x), \quad x \in \Omega, \\
\frac{\partial u^{\varepsilon}}{\partial \nu_{A}}=0, \quad x \in \partial \Omega,
\end{gathered}
$$


$\lambda>0$. Известно, что для любого $\varepsilon>0$ сушествует единственное решение $u^{\varepsilon} \in$ $H^{1}(\Omega)$ задачи (5.1). Изучим асимптотическое поведение функций $u^{\varepsilon}$ при $\varepsilon \rightarrow 0$.

ТЕОРема 5.1. Пусть выполнены условия теоремы 3.1. Тогда последовательность решений $u^{\varepsilon}=u^{\varepsilon}(x, \lambda)$ задачи $(5.1) D_{\mathscr{F}(\varepsilon)}$-сходится $\kappa$ решению $u=u(x, \lambda)$ следующей краевой задачи:

$$
\begin{gathered}
-\operatorname{div}\left(A^{*}(x) \nabla u\right)+\Phi_{f} \lambda \rho(x) u+b(x, \lambda) u=\rho(x) \lambda^{-1} f(x), \quad x \in \Omega, \\
\frac{\partial u}{\partial \nu_{A^{*}}}=0, \quad x \in \partial \Omega .
\end{gathered}
$$

ДокАЗАтЕльство. Хорошо известно, что решение $u^{\varepsilon}=u^{\varepsilon}(x, \lambda)$ задачи (5.1) минимизирует функционал

$$
J^{(\varepsilon)}[u]=\mu^{\varepsilon} \int_{\Omega}\left(\left(A^{\varepsilon}(x) \nabla u, \nabla u\right)+\lambda \Phi^{\varepsilon}|u|^{2}-2 \lambda^{-1} f^{\varepsilon} u\right) d x
$$

в классе функций $u \in H^{1}(\Omega)$. Поскольку $J^{(\varepsilon)}\left[u^{\varepsilon}\right] \leqslant J^{(\varepsilon)}[0]=0$, то в силу неравенства Коши и условия (C.5) имеем

$$
\mu^{\varepsilon} \int_{\Omega}\left(\left(A^{\varepsilon}(x) \nabla u^{\varepsilon}, \nabla u^{\varepsilon}\right)+\lambda \Phi^{\varepsilon}\left|u^{\varepsilon}\right|^{2}\right) d x \leqslant \frac{C}{\lambda} \mu^{\varepsilon}\left\|f^{\varepsilon}\right\|_{\mathscr{F}(\varepsilon)}^{2} \leqslant C .
$$

Из (5.5) и (1.8) следует, что

$$
\mu^{\varepsilon}\left\|u^{\varepsilon}\right\|_{1, \mathscr{F}(\varepsilon)} \leqslant C
$$

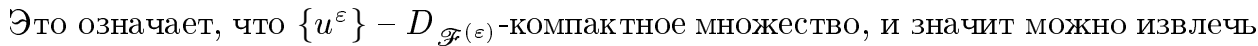
подпоследовательность (за которой мы сохраним обозначение $\left\{u^{\varepsilon}\right\}$ ), $D_{\mathscr{F}(\varepsilon)}$-сходяшуюся к некоторой функции $u \in L^{2}(\Omega)$. Покажем, что $u=u(x, \lambda)-$ решение задачи (5.2), (5.3). Доказательство этого факта будет проведено в два шага.

Шаг 1. Пусть $\eta$ - произвольная гладкая в $\Omega$ функция, и пусть $Y_{h}^{\varepsilon}(x), W_{M h}^{\varepsilon}(x)$, $G_{h}^{(\varepsilon)}$ - функции и множества, определенные в леммах 4.1 и 4.2. Покажем, что

$$
\varlimsup_{M \rightarrow \infty} \varlimsup_{h \rightarrow 0} \varlimsup_{\varepsilon \rightarrow 0} J^{(\varepsilon)}\left[\vartheta_{M h}^{\varepsilon}\right] \leqslant J_{b}[\eta],
$$

где $\vartheta_{M h}^{\varepsilon}(x)=Y_{h}^{\varepsilon}(x) W_{M h}^{\varepsilon}(x)$ и

$$
J_{b}[\eta]=\int_{\Omega}\left(\left(A^{*}(x) \nabla \eta, \nabla \eta\right)+\left(\Phi_{f} \lambda \rho(x)+b(x, \lambda)\right) \eta^{2}-2 \lambda^{-1} \rho(x) f(x) \eta\right) d x .
$$

Очевидно, имеем $\vartheta_{M h}^{\varepsilon} \in H^{1}(\Omega)$ и

$$
\begin{aligned}
J^{(\varepsilon)}\left[\vartheta_{M h}^{\varepsilon}\right]= & \mu^{\varepsilon} \int_{\Omega}\left(A^{\varepsilon}(x) \nabla \vartheta_{M h}^{\varepsilon}, \nabla \vartheta_{M h}^{\varepsilon}\right) d x \\
& +\mu^{\varepsilon} \int_{\Omega}\left(\lambda \Phi^{\varepsilon}\left|\vartheta_{M h}^{\varepsilon}\right|^{2}-2 \lambda^{-1} f^{\varepsilon} \vartheta_{M h}^{\varepsilon}\right) d x .
\end{aligned}
$$

Оценим правую часть (5.9). Из условий (C.1), (C.5) теоремы, утверждений (B), (C) леммы 4.1 и $(\mathrm{A})$ леммы 4.2 следует, что

$$
\lim _{M \rightarrow \infty} \lim _{h \rightarrow 0} \varlimsup_{\varepsilon \rightarrow 0} \mu^{\varepsilon} \int_{\Omega} f^{\varepsilon} \vartheta_{M h}^{\varepsilon} d x=\int_{\Omega} f \eta \rho(x) d x .
$$


Из утверждений (D) леммы 4.1 и (A) леммы 4.2 несложно также получить

$$
\mu^{\varepsilon} \int_{\Omega} \lambda \Phi^{\varepsilon}(x)\left|\vartheta_{M h}^{\varepsilon}\right|^{2} d x \leqslant \mu^{\varepsilon} \int_{\Omega} \lambda \Phi^{\varepsilon}(x) \eta^{2}(x)\left|Y_{h}^{\varepsilon}\right|^{2} d x+C M h(1+M h)
$$

при $h \rightarrow 0$.

Перепишем первый член в правой части (5.9) следующим образом:

$$
\begin{aligned}
\mu^{\varepsilon} \int_{\Omega}( & \left.A^{\varepsilon}(x) \nabla \vartheta_{M h}^{\varepsilon}, \nabla \vartheta_{M h}^{\varepsilon}\right) d x \\
= & \mu^{\varepsilon} \int_{\Omega}\left(A^{\varepsilon}(x) \nabla W_{M h}^{\varepsilon}, \nabla W_{M h}^{\varepsilon}\right)\left|Y_{h}^{\varepsilon}\right|^{2} d x \\
& +\mu^{\varepsilon} \int_{\Omega}\left(A^{\varepsilon}(x) \nabla Y_{h}^{\varepsilon}, \nabla Y_{h}^{\varepsilon}\right)\left|W_{M h}^{\varepsilon}\right|^{2} d x \\
& +2 \mu^{\varepsilon} \int_{\Omega}\left(A^{\varepsilon}(x) \nabla W_{M h}^{\varepsilon}, \nabla Y_{h}^{\varepsilon}\right) W_{M h}^{\varepsilon} Y_{h}^{\varepsilon} d x .
\end{aligned}
$$

Рассмотрим теперь первое слагаемое в правой части (5.12). Из (1.8), (1.9), утверждений $(\mathrm{A})$ леммы 4.1 и $(\mathrm{C})$ леммы 4.2 следует, что

$$
\varlimsup_{M \rightarrow \infty} \varlimsup_{h \rightarrow 0} \varlimsup_{\varepsilon \rightarrow 0} \mu^{\varepsilon} \int_{\Omega}\left(A^{\varepsilon}(x) \nabla W_{M h}^{\varepsilon}, \nabla W_{M h}^{\varepsilon}\right)\left|Y_{h}^{\varepsilon}\right|^{2} d x \leqslant \int_{\Omega}\left(A^{*}(x) \nabla \eta, \nabla \eta\right) d x .
$$

Оценим второе слагаемое в правой части (5.12). Из утверж дений (D) леммы 4.1 и (А) леммы 4.2 следует неравенство

$$
\begin{aligned}
& \mu^{\varepsilon} \int_{\Omega}\left(A^{\varepsilon}(x) \nabla Y_{h}^{\varepsilon}, \nabla Y_{h}^{\varepsilon}\right)\left|W_{M h}^{\varepsilon}\right|^{2} d x \\
& \quad \leqslant \mu^{\varepsilon} \int_{\Omega}\left(A^{\varepsilon}(x) \nabla Y_{h}^{\varepsilon}, \nabla Y_{h}^{\varepsilon}\right) \eta^{2} d x+C M h(1+M h) .
\end{aligned}
$$

Рассмотрим, наконец, третье слагаемое в правой части (5.12). Из (1.8), (1.9), утверждений (A)-(D) леммы 4.1 и (A), (В) леммы 4.2 имеем

$$
\lim _{h \rightarrow 0} \varlimsup_{\varepsilon \rightarrow 0} \mu^{\varepsilon} \int_{\Omega}\left|\left(A^{\varepsilon}(x) \nabla W_{M h}^{\varepsilon}, \nabla Y_{h}^{\varepsilon}\right)\right|\left|W_{M h}^{\varepsilon}\right| Y_{h}^{\varepsilon} d x=0 .
$$

Тогда из (5.10)-(5.15) и утвеждения (D) леммы 4.1 получаем (5.7). Поскольку $u^{\varepsilon}$ минимизирует функционал (5.4), из (5.7) следует, что

$$
\varlimsup_{\varepsilon \rightarrow 0} J^{(\varepsilon)}\left[u^{\varepsilon}\right] \leqslant J_{b}[\eta]
$$

для любой гладкой функции $\eta$. Очевидно, что (5.16) вьполняется и для любой функции $\eta \in H^{1}(\Omega)$.

Шаг 2. Пусть $\left\{u^{\varepsilon}\right\}$ - некоторая последовательность решений задачи (5.1), $D_{\mathscr{F}(\varepsilon)}$-сходящаяся к функции $u$. Покажем, что

$$
\varliminf_{\varepsilon \rightarrow 0} J^{(\varepsilon)}\left[u^{\varepsilon}\right] \geqslant J_{b}[u] .
$$

Согласно лемме 4.4 сушествует семейство функций $\left\{u_{M}^{\varepsilon} \in \operatorname{Lip}(C M, \Omega)\right.$, $M=1,2, \ldots\}$ таких, что

$$
\lim _{M \rightarrow \infty} \varlimsup_{\varepsilon \rightarrow 0} \mu^{\varepsilon}\left\|u^{\varepsilon}-u_{M}^{\varepsilon}\right\|_{\mathscr{F}(\varepsilon)}=0 .
$$


Кроме того, для любого $M$ найдется подпоследовательность $\left\{u_{M}^{\varepsilon_{j}}\right\}, \varepsilon_{j} \rightarrow 0$, сходяшаяся равномерно в $\Omega$ к некоторой функции $u_{M}$, и

$$
\underline{\lim }_{\varepsilon=\varepsilon_{j} \rightarrow 0} \mu^{\varepsilon} \int_{\mathscr{F}(\varepsilon)}\left(A^{\varepsilon}(x) \nabla u^{\varepsilon}, \nabla u^{\varepsilon}\right) d x \geqslant \varlimsup_{M \rightarrow \infty} \int_{\Omega}\left(A^{*}(x) \nabla u_{M}, \nabla u_{M}\right) d x-\xi(M),
$$

где $\xi(M) \rightarrow 0$ при $M \rightarrow \infty$. Поскольку последовательность $\left\{u^{\varepsilon}\right\} D_{\mathscr{F}(\varepsilon) \text {-сходится }}$ к $u$, a $\left\{u_{M}^{\varepsilon_{j}}\right\}$ сходится равномерно к $u_{M}$, из (5.18) следует, что функции $u_{M}$ сходятся в $L^{2}(\Omega)$ к $u$ при $M \rightarrow \infty$. Вместе с этим вследствие (5.19) последовательность $\left\{u_{M}, M=1,2, \ldots\right\}$ ограничена в $H^{1}(\Omega)$ (тензор $A^{*}(x)$ положительно определен). Таким образом, $u \in H^{1}(\Omega)$.

Аппроксимируем $u$ гладкими в $\Omega$ функциями $u_{\delta}, \delta>0$,

$$
\left\|u_{\delta}-u\right\|_{1, \Omega}^{2} \leqslant \delta
$$

и положим $w_{\delta}(x)=u_{\delta}(x)-u(x)$. В силу леммы 4.3 сушествует последовательность $\left\{w_{\delta}^{\varepsilon}\right\}, D_{\mathscr{F}(\varepsilon)}$-сходящаяся к $w_{\delta}$ и такая, что

$$
\varlimsup_{\varepsilon \rightarrow 0} I^{(\varepsilon)}\left[w_{\delta}^{\varepsilon}\right] \leqslant C \delta
$$

где $C$ - постоянная, которая не зависит от $u_{\delta}$, а функционал $I^{(\varepsilon)}$ определен в (4.15).

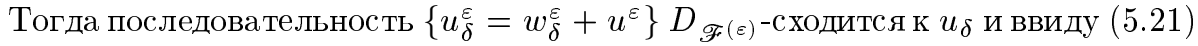

$$
\lim _{\varepsilon \rightarrow 0} J^{(\varepsilon)}\left[u_{\delta}^{\varepsilon}\right] \leqslant \lim _{\varepsilon \rightarrow 0} J^{(\varepsilon)}\left[u^{\varepsilon}\right]+C \delta,
$$

где постоянная $C$ не зависит от $u_{\delta}$ (переходя в случае необходимости к подпоследовательности, мы можем предполагать, что предел в левой части (5.22) существует). Поскольку $u_{\delta}(x)$ - гладкая в $\Omega$ функция, имеем

$$
\lim _{\varepsilon \rightarrow 0} \mu^{\varepsilon} \int_{\mathscr{F}(\varepsilon)}\left|u_{\delta}^{\varepsilon}-u_{\delta}\right|^{2} d x=0 .
$$

Из неравенства (5.22) и леммы 4.4 следует, что существуют функции $u_{\delta M}^{\varepsilon} \in \operatorname{Lip}(\mathrm{C} M, \Omega), M=1,2, \ldots$, и множества $G_{M}^{(\varepsilon)}$ такие, что $u_{\delta M}^{\varepsilon}(x)=u_{\delta}^{\varepsilon}(x)$ для $x \in \mathscr{F}(\varepsilon) \backslash G_{M}^{(\varepsilon)}$ и $\mu^{\varepsilon}$ meas $G_{M}^{(\varepsilon)}=o\left(1 / M^{2}\right), \mu^{\varepsilon}\left\|u_{\delta}^{\varepsilon}\right\|_{G_{M}^{(\varepsilon)}}^{2}=o(1)$ для достаточно малых $\varepsilon$ и $M \rightarrow \infty$. Кроме того, для каждого фиксированного $M \in\{1,2, \ldots\}$ можно выделить подпоследовательность $u_{\delta M}^{\varepsilon_{j}}, \varepsilon_{j} \rightarrow 0$, равномерно в $\Omega$ сходяшуюся к некоторой функции $u_{\delta} \in \operatorname{Lip}(C M, \Omega)$. В то же время, функции $u_{\delta} M$ сходятся в пространтсве $L^{2}(\Omega)$ к $u_{\delta}$ при $M \rightarrow \infty$, поскольку

$$
\begin{aligned}
& \int_{\Omega}\left|u_{\delta M}-u_{\delta}\right|^{2} d x \\
& \quad \leqslant C \varlimsup_{\varepsilon=\varepsilon_{j} \rightarrow 0} \mu^{\varepsilon} \int_{\mathscr{F}(\varepsilon)}\left(\left|u_{\delta M}-u_{\delta M}^{\varepsilon}\right|^{2}+\left|u_{\delta M}^{\varepsilon}-u_{\delta}^{\varepsilon}\right|^{2}+\left|u_{\delta}^{\varepsilon}-u_{\delta}\right|^{2}\right) d x \underset{M \rightarrow \infty}{\longrightarrow} 0 .
\end{aligned}
$$

Из (5.23) следует, что существует последовательность $\left\{r^{\varepsilon}>0\right\}, r^{\varepsilon} \rightarrow 0$, и множества $B_{M}^{(\varepsilon)}$ такие, что

$$
\begin{gathered}
\lim _{\varepsilon \rightarrow 0} \mu^{\varepsilon} \text { meas } B_{M}^{(\varepsilon)}=0, \\
\left|u_{\delta M}^{\varepsilon}(x)-u_{\delta}(x)\right| \leqslant r^{\varepsilon}, \quad x \in \mathscr{F}^{(\varepsilon)} \backslash \widetilde{G}_{M}^{(\varepsilon)},
\end{gathered}
$$


где $\widetilde{G}_{M}^{(\varepsilon)}=G_{M}^{(\varepsilon)} \cup B_{M}^{(\varepsilon)}$. Определим функции

$$
v_{\delta M}^{\varepsilon}(x)= \begin{cases}u_{\delta}(x)+r^{\varepsilon}, & u_{\delta M}^{\varepsilon}(x)>u_{\delta}(x)+r^{\varepsilon} \\ u_{\delta M}^{\varepsilon}(x), & \left|u_{\delta M}^{\varepsilon}(x)-u_{\delta}(x)\right| \leqslant r^{\varepsilon} \\ u_{\delta}(x)-r^{\varepsilon}, & u_{\delta M}^{\varepsilon}(x)<u_{\delta}(x)-r^{\varepsilon}\end{cases}
$$

Ясно, что $v_{\delta M}^{\varepsilon} \in \operatorname{Lip}(C M, \Omega)$ при достаточно больших $M$. Кроме того, функции $v_{\delta M}^{\varepsilon}$ равномерно в $\Omega$ сходятся к $u_{\delta}$ при $\varepsilon \rightarrow 0$.

Поскольку $v_{\delta M}^{\varepsilon}(x)=u_{\delta}^{\varepsilon}(x)$ для $x \in \mathscr{F}(\varepsilon) \backslash \widetilde{G}_{M}^{(\varepsilon)}$, имеем

$$
\begin{aligned}
I^{(\varepsilon)}\left[u_{\delta}^{\varepsilon}\right]= & \mu^{\varepsilon}\left(\int_{\mathscr{F}(\varepsilon)}\left(A^{\varepsilon}(x) \nabla \widetilde{v}_{\delta M}^{\varepsilon}, \nabla \widetilde{v}_{\delta M}^{\varepsilon}\right) d x\right. \\
& \left.+\int_{\mathscr{M}^{(\varepsilon)}}\left(\left(A^{\varepsilon}(x) \nabla u_{\delta}^{\varepsilon}, \nabla u_{\delta}^{\varepsilon}\right)+\lambda \Phi_{m}\left|u_{\delta}^{\varepsilon}\right|^{2}\right) d x\right) \\
& +\mu^{\varepsilon}\left(\int_{\mathscr{F}(\varepsilon) \backslash \widetilde{G}_{M}^{(\varepsilon)}}\left(A^{\varepsilon}(x) \nabla u_{\delta}^{\varepsilon}, \nabla u_{\delta}^{\varepsilon}\right) d x+\lambda \int_{\mathscr{F}(\varepsilon)} \Phi_{\mathscr{F}}\left|u_{\delta}^{\varepsilon}\right|^{2} d x\right) \\
& +\mu^{\varepsilon} \int_{\mathscr{F}(\varepsilon) \cap \widetilde{G}_{M}^{\varepsilon}}\left(2\left(A^{\varepsilon}(x) \nabla u_{\delta}^{\varepsilon}, \nabla v_{\delta M}^{\varepsilon}\right)-\left(A^{\varepsilon}(x) \nabla v_{\delta M}^{\varepsilon}, \nabla v_{\delta M}^{\varepsilon}\right)\right) d x \\
\equiv & \Xi_{1}+\Xi_{2}+\Xi_{3},
\end{aligned}
$$

где $\widetilde{v}_{\delta M}^{\varepsilon}=u_{\delta}^{\varepsilon}-v_{\delta}^{\varepsilon}$. Оценим снизу каждое из слагаемых $\Xi_{i}$ в $(5.24)$.

Рассмотрим подобласть $\Omega_{\zeta}=\left\{x \in \Omega:\left|u_{\delta}(x)\right|>2 \zeta\right\}$ области $\Omega$, где $\zeta>0$. Покроем $\Omega_{\zeta}$ кубами $K_{h}^{\alpha}=\left\{x:\left|x_{l}-x_{l}^{\alpha}\right| \leqslant h / 2\right\}$, непересекаюшимися во внутренних точках. Для достаточно малых $\varepsilon$ и $h$ имеем $\left|v_{\delta}^{\varepsilon}(x)\right|>\zeta$, когда $x \in K_{h}^{\alpha}$, и после простых вычислений, используя (1.9), получаем

$$
\begin{aligned}
& \left(1+\frac{1}{\eta}\right)\left(A^{\varepsilon}(x) \nabla\left(u_{\delta}^{\varepsilon}-v_{\delta M}^{\varepsilon}\right), \nabla\left(u_{\delta}^{\varepsilon}-v_{\delta M}^{\varepsilon}\right)\right) \\
& \quad \geqslant\left|v_{\delta M}^{\varepsilon}\right|^{2}\left(A^{\varepsilon}(x) \nabla \frac{u_{\delta}^{\varepsilon}}{v_{\delta M}^{\varepsilon}}, \nabla \frac{u_{\delta}^{\varepsilon}}{v_{\delta M}^{\varepsilon}}\right)-(1+\eta)\left|\frac{\nabla v_{\delta M}^{\varepsilon}}{v_{\delta M}^{\varepsilon}}\right|^{2}\left|u_{\delta}^{\varepsilon}-v_{\delta M}^{\varepsilon}\right|^{2} C_{2}
\end{aligned}
$$

при $x \in \mathscr{F}^{(\varepsilon)} \cap K_{h}^{\alpha}$ и

$$
\begin{aligned}
& \left(1+\frac{1}{\eta}\right)\left(A^{\varepsilon}(x) \nabla u_{\delta}^{\varepsilon}, \nabla u_{\delta}^{\varepsilon}\right) \\
& \quad \geqslant\left|v_{\delta M}^{\varepsilon}\right|^{2}\left(A^{\varepsilon}(x) \nabla \frac{u_{\delta}^{\varepsilon}}{v_{\delta M}^{\varepsilon}}, \nabla \frac{u_{\delta}^{\varepsilon}}{v_{\delta M}^{\varepsilon}}\right)-(1+\eta)\left|u_{\delta}^{\varepsilon} \frac{\nabla v_{\delta M}^{\varepsilon}}{v_{\delta M}^{\varepsilon}}\right|^{2} c_{2}^{\varepsilon}
\end{aligned}
$$

при $x \in \mathscr{M}^{(\varepsilon)} \cap K_{h}^{\alpha}$. В этих неравенствах $\eta$ - произвольная положительная постоянная. Воспользуемся теперь соотношением (5.23), определением и свойствами функции $v_{\delta M}^{\varepsilon}(x)$. Полагая $\eta=1 / h$ в (5.25), (5.26), для любого $K_{h}^{\alpha} \subset \Omega_{\zeta}$ получаем

$$
\begin{aligned}
& \mu^{\varepsilon} \int_{K_{h}^{\alpha} \cap \mathscr{F}(\varepsilon)}\left(A^{\varepsilon}(x) \nabla \widetilde{v}_{\delta M}^{\varepsilon}, \nabla \widetilde{v}_{\delta M}^{\varepsilon}\right) d x \\
& +\mu^{\varepsilon} \int_{K_{h}^{\alpha} \cap \mathscr{M}^{(\varepsilon)}}\left(\left(A^{\varepsilon}(x) \nabla u_{\delta}^{\varepsilon}, \nabla u_{\delta}^{\varepsilon}\right)+\lambda \Phi \mathscr{M}\left|u_{\delta}^{\varepsilon}\right|^{2}\right) d x \\
& \geqslant\left|u_{\delta}\left(x^{\alpha}\right)\right|^{2} \mu^{\varepsilon}\left(\int_{K_{h}^{\alpha}}\left(A^{\varepsilon}(x) \nabla \frac{u_{\delta}^{\varepsilon}}{v_{\delta M}^{\varepsilon}}, \nabla \frac{u_{\delta}^{\varepsilon}}{v_{\delta M}^{\varepsilon}}\right) d x\right. \\
& \left.\quad+\lambda \int_{K_{h}^{\alpha} \cap \mathscr{M}^{(\varepsilon)}} \Phi{ }_{\mathscr{M}}\left(\frac{u_{\delta}^{\varepsilon}}{v_{\delta M}^{\varepsilon}}\right)^{2} d x\right)+o\left(h^{n}\right)
\end{aligned}
$$


при достаточно малых $\varepsilon(\varepsilon \leqslant \widehat{\varepsilon}(h))$ и $h \rightarrow 0$. Кроме того, используя условие (С.4), ВЫводим

$$
\begin{aligned}
\lim _{\varepsilon \rightarrow 0} \mu^{\varepsilon} & \left(\int_{K_{h}^{\alpha}}\left(A^{\varepsilon}(x) \nabla \frac{u_{\delta}^{\varepsilon}}{v_{\delta M}^{\varepsilon}}, \nabla \frac{u_{\delta}^{\varepsilon}}{v_{\delta M}^{\varepsilon}}\right) d x\right. \\
& \left.+\lambda \int_{K_{h}^{\alpha} \cap \mathscr{M}^{(\varepsilon)}} \Phi \mathscr{M}\left(\frac{u_{\delta}^{\varepsilon}}{v_{\delta M}^{\varepsilon}}\right)^{2} d x\right) \geqslant h^{n} b\left(x^{\alpha}, \lambda\right)+o\left(h^{n}\right)
\end{aligned}
$$

при $h \rightarrow 0$. Из (5.27), (5.28) следует, что

$$
\lim _{\varepsilon \rightarrow 0} \Xi_{1} \geqslant \int_{\Omega_{\zeta}} b(x, \lambda)\left|u_{\delta}\right|^{2} d x
$$

Пользуясь определением подобласти $\Omega_{\zeta}$ и перходя к пределу при $\zeta \rightarrow 0$ в $(5.29)$, получаем

$$
\lim _{\varepsilon \rightarrow 0} \Xi_{1} \geqslant \int_{\Omega} b(x, \lambda)\left|u_{\delta}\right|^{2} d x
$$

Для получения оценки снизу для слагаемого $\Xi_{2}$ в (5.24) мы поступаем следуюшим образом. Применяя лемму 4.4 и используя (5.23) и условие (C.1), получаем

$$
\underline{\lim }_{\varepsilon=\varepsilon_{j} \rightarrow 0} \Xi_{2} \geqslant \int_{\Omega}\left(A^{*}(x) \nabla u_{\delta M}, \nabla u_{\delta M}\right) d x+\int_{\Omega} \lambda \Phi_{\mathscr{F}}\left|u_{\delta}\right|^{2} \rho(x) d x-\xi(M)
$$

где $\xi(M) \rightarrow 0$ при $M \rightarrow \infty$. Поскольку первое слагаемое в правой части $(5.31)$ является слабо полунепрерьвньм снизу функционалом в $H^{1}(\Omega)$ и функции $u_{\delta} M$ сходятся в $L^{2}(\Omega)$ к $u_{\delta}$ при $M \rightarrow \infty$, имеем

$$
\underline{\lim }_{M \rightarrow \infty} \underset{\varepsilon=\varepsilon_{j} \rightarrow 0}{\lim } \Xi_{2} \geqslant \int_{\Omega}\left(\left(A^{*}(x) \nabla u_{\delta}, \nabla u_{\delta}\right)+\lambda \Phi_{\mathscr{F}} \rho(x)\left|u_{\delta}\right|^{2}\right) d x .
$$

Наконец, для последнего слагаемого в правой части (5.24) легко получаем

$$
\lim _{M \rightarrow \infty} \varlimsup_{\varepsilon \rightarrow 0}\left|\Xi_{3}\right|=0
$$

Таким образом, из (5.30), (5.32), (5.33) и (5.23) с учетом условий (C.1), (С.5) теоремы 3.1 имеем

$$
\varliminf_{\varepsilon \rightarrow 0} J^{(\varepsilon)}\left[u_{\delta}^{\varepsilon}\right] \geqslant J_{b}\left[u_{\delta}\right]
$$

Из этого неравенства, (5.20) и (5.22) немедленно следует (5.17).

Неравенства $(5.16),(5.17)$ означают, что если некоторая подпоследовательность решений задачи (5.1) $D_{\mathscr{F}(\varepsilon)}$-сходится к функции $u=u(x, \lambda)$, то $u$ минимизирует функционал (5.8) в пространстве $H^{1}(\Omega)$, т.е. $u(x, \lambda)$ - решение задачи $(5.2),(5.3)$. Поскольку $\lambda>0$ и $b(x, \lambda) \geqslant 0$, эта задача имеет единственное решение и вся последовательность решений задачи $(5.1) D_{\mathscr{F}(\varepsilon)}$-сходится к функции $u=u(x, \lambda)$. Теорема 5.1 доказана. 


\section{§6. Доказательство теоремы 3.1}

Рассмотрим краевую задачу (5.1) при комплексных $\lambda$ таких, что $\arg \lambda \neq \pi$. Известно, что решение $u^{\varepsilon}(x, \lambda)$ задачи (5.1) является аналитической по $\lambda$ функцией в разрезанной комплексной плоскости $\mathbb{C} \backslash\{\arg \lambda=\pi\}$, и из условия (С.5) следует, что

$$
\left\|u^{\varepsilon}(\cdot, \lambda)\right\|_{\mathscr{F}(\varepsilon)}^{2} \leqslant \frac{C}{|\lambda|^{4}} \text { meas } \mathscr{F}^{(\varepsilon)}, \quad|\arg \lambda-\pi| \geqslant \theta_{0}>0 .
$$

Кроме того, решение $u^{\varepsilon}(x, t)$ задачи (1.4)-(1.6) может быть представлено с помощью обратного преобразвания Лапласа в следующем виде:

$$
u^{\varepsilon}(x, t)=\frac{1}{2 \pi i} \int_{\sigma-i \infty}^{\sigma+i \infty} e^{\lambda t} u^{\varepsilon}(x, \lambda) d \lambda, \quad \sigma>0
$$

где $u^{\varepsilon}(x, \lambda)$ - решение задачи (5.1). С другой стороны, стандартным образом (см., например, [17]) легко получить оценку

$$
\max _{t \in[0, T]}\left\|u^{\varepsilon}(\cdot, t)\right\|_{1, \mathscr{F} \varepsilon}^{2}+\int_{0}^{T}\left\|u_{t}^{\varepsilon}(\cdot, t)\right\|_{\Omega}^{2} d t \leqslant C(T) \text { meas } \mathscr{F}^{(\varepsilon)} .
$$

Из этого неравенства следует, что для любого $t \in(0, T)\left\{u^{\varepsilon}\right\}-D_{\mathscr{F} \varepsilon \text {-компактное }}$ множество и сушествует подпоследовательность, за которой сохраним обозначение

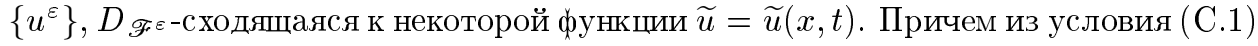
вытекает, что

$$
\lim _{\varepsilon \rightarrow 0} \mu^{\varepsilon} \int_{\mathscr{F}(\varepsilon)} u^{\varepsilon}(x, t) \varphi(x) d x=\int_{\Omega} \widetilde{u}(x, t) \varphi(x) \rho(x) d x
$$

для любой функции $\varphi \in C(\Omega)$. Покажем, что $\widetilde{u}(x, t)$ - решение задачи $(3.4)-(3.6)$.

Из (6.1), (6.2) следует, что

$$
\mu^{\varepsilon} \int_{\mathscr{F}(\varepsilon)} u^{\varepsilon}(x, t) \varphi(x) d x=\frac{1}{2 \pi i} \int_{\sigma-i \infty}^{\sigma+i \infty} e^{\lambda t} F_{\lambda}^{(\varepsilon)}[\varphi] d \lambda
$$

где

$$
F_{\lambda}^{(\varepsilon)}[\varphi]=\mu^{\varepsilon} \int_{\mathscr{F}(\varepsilon)} u^{\varepsilon}(x, \lambda) \varphi(x) d x .
$$

В силу теоремы 5.1 для любого $\lambda>0$ имеем

$$
\lim _{\varepsilon \rightarrow 0} F_{\lambda}^{(\varepsilon)}[\varphi]=\int_{\Omega} u(x, \lambda) \varphi(x) \rho(x) d x,
$$

где $u(x, \lambda)$ - решение задачи $(5.2),(5.3)$. Ввиду $(6.1)$ функционалы $F_{\lambda}^{(\varepsilon)}: C(\Omega) \rightarrow \mathbb{C}$ равномено ограничены по $\lambda$ на любом компактном подомножестве области $\Gamma_{\theta_{0}}=$ $\left\{\lambda:|\arg \lambda-\pi|>\theta_{0}\right\}, \theta_{0}>0$, и зависят аналитически от параметра $\lambda \in \Gamma_{\theta_{0}}$. Следовательно, согласно теореме Витали последовательность $\left\{F_{\lambda}^{(\varepsilon)}\right\}$ сходится по норме к некоторому функционалу $F_{\lambda}$ равномерно по $\lambda$ на любом компактном подомножестве области $\Gamma_{\theta_{0}}$. Причем функционал $F_{\lambda}$ аналитически зависит от параметра $\lambda$ и в силу оценки (6.1)

$$
\left\|F_{\lambda}[\varphi]\right\| \leqslant \frac{C}{|\lambda|^{2}}\|\varphi\|_{\Omega}
$$


Таким образом, вследстие теоремы Рисса и равенства (6.6) представление

$$
F_{\lambda}[\varphi]=\int_{\Omega} u(x, \lambda) \varphi(x) \rho(x) d x
$$

имеет место длю любого $\lambda \in\left\{\lambda:|\arg \lambda-\pi| \geqslant \theta_{0}>0\right\}$, причем $u(x, \lambda)$ - аналитическая по $\lambda$ функция, удовлетворяющая оценке

$$
\|u(\cdot, \lambda)\|_{\Omega}^{2} \leqslant \frac{C}{|\lambda|^{4}}, \quad \lambda \in \Gamma_{\theta_{0}}
$$

Таким образом, соотношение (6.6) справедливо для любого $\lambda \in \mathbb{C} \backslash\{\arg \lambda=\pi\}$.

Перейдем теперь в краевой задаче $(5.2),(5.3)$ к комплексным $\lambda$. Для этого воспользуемся следующим результатом, который доказывается таким же образом, как лемма 3 в работе [23].

ЛЕмма 6.1. Функиия $b(x, \lambda)$, определенная в условии (С.4) для $\lambda>0$, имеет аналитическое по $\lambda$ продолжение $\widetilde{b}(x, \lambda)$ в разрезанную комплексную плоскость $\mathbb{C} \backslash\{\arg \lambda=\pi\}$, причем $\widetilde{b}(x, \lambda)=O\left(|\lambda|^{\delta}\right), \delta<1$, nрu $|\lambda| \rightarrow \infty u$ $|\arg \lambda-\pi| \geqslant \theta_{0}>0$.

Продолжим функцию $b(x, \lambda)$, полагая $b(x, \lambda)=\widetilde{b}(x, \lambda)$ для $\lambda \in \mathbb{C} \backslash \mathbb{R}$, и рассмотрим задачу (5.2), (5.3) при $\lambda \in \mathbb{C} \backslash\{\arg \lambda=\pi\}$. Функция $u(x, \lambda)$ является решением (5.2), (5.3) при вешественных $\lambda>0$, она аналитична по $\lambda \in \Gamma_{\theta_{0}}$ (для любого $\left.\theta_{0}>0\right)$ и удовлетворяет (6.7). Поскольку тензор $A^{*}(x)$ положительно определен, $b(x, \lambda) \geqslant 0$ при $\lambda>0$ и $b(x, \lambda)$ - аналитическая по $\lambda$ функция в разрезанной комплексной плоскости $\mathbb{C} \backslash\{\arg \lambda=\pi\}$, то краевая задача $(5.2)-(5.3)$ имеет единственное решение $u(x, \lambda)$ для $\lambda \in \mathbb{C} \backslash\{\arg \lambda=\pi\}$.

Заметим, что вследствие леммы 6.1 формула (3.7) действительно определяет локально интегрируемую по $t \geqslant 0$ функцию $B(x, t)$, которая не зависит от $\sigma>0$. Тогда решение $u(x, t)$ задачи (3.4)-(3.6) может быть представлено в виде

$$
u(x, t)=\frac{1}{2 \pi i} \int_{\sigma-i \infty}^{\sigma+i \infty} e^{\lambda t} u(x, \lambda) d \lambda, \quad \sigma>0
$$

и из (6.7), (6.8) следует, что

$$
\int_{\Omega} u(x, t) \rho(x) \varphi(x) d x=\frac{1}{2 \pi i} \int_{\sigma-i \infty}^{\sigma+i \infty} e^{\lambda t}\left(\int_{\Omega} u(x, \lambda) \rho(x) \varphi(x) d x\right) d \lambda
$$

Тогда из $(6.1),(6.5)-(6.7)$ и (6.9) получаем

$$
\lim _{\varepsilon \rightarrow 0} \int_{\Omega} u^{\varepsilon}(x, t) \mathbb{I}_{f}^{\varepsilon} \varphi(x) d x=\int_{\Omega} u(x, t) \rho(x) \varphi(x) d x
$$

Наконец, в силу (6.4) и (6.10) мы заключаем, что $\widetilde{u}(x, t)=u(x, t)$. Теорема 3.1 доказана. 


\section{§ 7. Периодическая модель двойной пористости с тонкими трещинами}

Пусть $\Omega=\mathscr{F}(\varepsilon) \cup \mathscr{M}^{(\varepsilon)} \cup \partial \mathscr{M}^{(\varepsilon)}$ - ограниченная область в $\mathbb{R}^{3}$ с кусочно-гладкой границей $\partial \Omega$. Будем предполагать, что система трещин $\mathscr{F}(\varepsilon)$, т.е. материал с высокой проводимостью, распределен в тонких ортогональных слоях толщины

$$
d_{\varepsilon}=d \varepsilon^{\theta / 2}, \quad d>0, \quad \theta>2,
$$

а матрица $\mathscr{M}^{(\varepsilon)}$ состоит из слабопроводящих кубических блоков $\mathscr{M}_{i}^{(\varepsilon)}$ с центрами в точках $x^{i, \varepsilon} \in \Omega$. Центры кубов $x^{i, \varepsilon}$ периодически, с периодом $\varepsilon$, распределены в $\Omega$. Рассмотрим начально-краевую задачу (1.4)-(1.6), где $A^{\varepsilon}(x)=a^{\varepsilon}(x) I, I-$ единичная матрица и

$$
a^{\varepsilon}(x)=a \varepsilon^{\theta} \cdot \mathbb{I}_{m}^{\varepsilon}(x)+1 \cdot \mathbb{I}_{f}^{\varepsilon}(x) .
$$

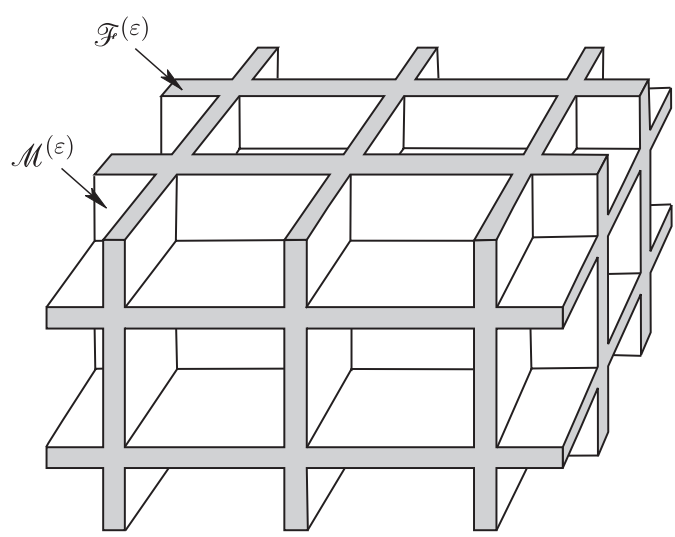

Рис. 1

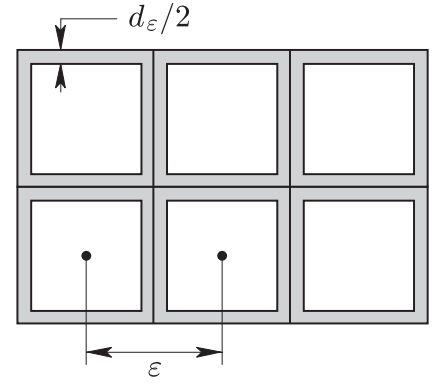

Рис. 2

Покажем, что в этом случае условия теоремы 3.1 выполнены и получим коэффициенты усредненной модели в явном виде. Прежде всего ясно, что

$$
\text { meas } \mathscr{F}^{(\varepsilon)}=\frac{\text { meas } \Omega}{\varepsilon^{3}}\left(\varepsilon^{3}-\left(\varepsilon-d_{\varepsilon}\right)^{3}\right)=3 \frac{d_{\varepsilon}}{\varepsilon} \text { meas } \Omega+o\left(\frac{d_{\varepsilon}}{\varepsilon}\right)
$$

и, следовательно,

$$
\mu^{\varepsilon} \simeq \frac{1}{3} \frac{\varepsilon}{d_{\varepsilon}}=\frac{1}{3 d} \varepsilon^{(2-\theta) / 2} .
$$

Легко видеть теперь, что условие (C.1) теоремы 3.1 выполнено и

$$
\rho(x)=1 \text {. }
$$

Далее, как и в [24] (см. также [18]), можно показать, что семейство множеств $\mathscr{F}(\varepsilon)$ удовлетворяет $S C$-условию, условие (С.3) теоремы 3.1 выполнено и

$$
a_{i j}^{*}(x)=\frac{2}{3} \delta_{i j},
$$

где $\delta_{i j}$ - символ Кронекера. 
Остается проверить условие (С.4). Для этого рассмотрим краевую задачу

$$
\begin{gathered}
\Delta W^{\varepsilon}-\lambda \Phi_{m} \beta_{\varepsilon} W^{\varepsilon}=0, \quad x \in \mathscr{M}, \\
W^{\varepsilon}=1, \quad x \in \partial \mathscr{M},
\end{gathered}
$$

где $\lambda>0$,

$$
\beta^{\varepsilon}=\frac{\left(\varepsilon-d_{\varepsilon}\right)^{2}}{a \varepsilon^{\theta}} \simeq \frac{1}{a} \varepsilon^{2-\theta}
$$

и $\mathscr{M}=\left\{x \in \mathbb{R}^{3}:\left|x_{i}\right|<1 / 2\right\}$.

В нашем случае функционал (3.3) имеет вид

$$
b_{\lambda}^{\varepsilon, h}(z)=\inf _{w^{\varepsilon}} \mu^{\varepsilon} \int_{K_{h}^{z}}\left(a^{\varepsilon}(x)\left|\nabla w^{\varepsilon}\right|^{2}+\lambda \Phi_{m} \mathbb{I}_{m}^{\varepsilon}\left|w^{\varepsilon}\right|^{2}+h^{-2-\gamma} \mathbb{I}_{f}^{\varepsilon}\left|w^{\varepsilon}-1\right|^{2}\right) d x
$$

где инфимум берется в классе функций $v \in H^{1}\left(K_{h}^{z}\right)$. Будем искать функцию $w^{\varepsilon}(x)$, минимизирующую (7.10), в виде

$$
w^{\varepsilon}(x)=\vartheta^{\varepsilon}(x)+\zeta^{\varepsilon}(x),
$$

где

$$
\vartheta^{\varepsilon}(x)= \begin{cases}W^{\varepsilon}\left(\frac{x-x^{i, \varepsilon}}{\varepsilon-d_{\varepsilon}}\right), & x \in \mathscr{M}_{i}^{(\varepsilon)} \cap K_{h}^{z} ; \\ 1, & x \in \mathscr{F}^{(\varepsilon)} \cap K_{h}^{z} .\end{cases}
$$

Тогда имеем

$$
\begin{aligned}
b_{\lambda}^{\varepsilon, h}(z)= & \mu^{\varepsilon} \int_{K_{h}^{z}}\left(a^{\varepsilon}(x)\left|\nabla \vartheta^{\varepsilon}\right|^{2}+\lambda \Phi_{m} \mathbb{I}_{m}^{\varepsilon}\left|\vartheta^{\varepsilon}\right|^{2}\right) d x \\
& +\mu^{\varepsilon} \int_{K_{h}^{z} \cap \mathscr{M}^{(\varepsilon)}}\left(a \varepsilon^{\theta}\left|\nabla \zeta^{\varepsilon}\right|^{2}+\lambda \Phi_{m}\left|\zeta^{\varepsilon}\right|^{2}\right) d x \\
& +\mu^{\varepsilon} \int_{K_{h}^{z} \cap \mathscr{F}(\varepsilon)}\left(\left|\nabla \zeta^{\varepsilon}\right|^{2}+h^{-2-\gamma}\left|\zeta^{\varepsilon}\right|^{2}\right) d x \\
& +2 \mu^{\varepsilon} \int_{K_{h}^{z} \cap \mathscr{M}^{(\varepsilon)}}\left(a \varepsilon^{\theta}\left(\nabla \vartheta^{\varepsilon}, \nabla \zeta^{\varepsilon}\right)+\lambda \vartheta^{\varepsilon} \zeta^{\varepsilon}\right) d x .
\end{aligned}
$$

Покажем, что функция $\zeta^{\varepsilon}(x)$ дает исчезающий вклад (при $\varepsilon \rightarrow 0, h \rightarrow 0$ ) в (7.10). Из (7.7), (7.8), (7.12) и (7.13) следует, что $\zeta^{\varepsilon}$ минимизирует функционал

$$
\begin{aligned}
I^{(\varepsilon)}\left[\zeta^{\varepsilon}\right]= & \mu^{\varepsilon} \int_{K_{h}^{z} \cap \mathscr{M}^{(\varepsilon)}}\left(a \varepsilon^{\theta}\left|\nabla \zeta^{\varepsilon}\right|^{2}+\lambda \Phi_{m}\left|\zeta^{\varepsilon}\right|^{2}\right) d x \\
& +\mu^{\varepsilon} \int_{K_{h}^{z} \cap \mathscr{F}(\varepsilon)}\left(\left|\nabla \zeta^{\varepsilon}\right|^{2}+h^{-2-\gamma}\left|\zeta^{\varepsilon}\right|^{2}\right) d x \\
& +2 \mu^{\varepsilon} \int_{K_{h}^{z} \cap \partial \mathscr{M}^{(\varepsilon)}} a^{\varepsilon}(x) \frac{\partial \vartheta^{\varepsilon}}{\partial \nu} \zeta^{\varepsilon} d \sigma .
\end{aligned}
$$


Поскольку $I^{(\varepsilon)}[0]=0$, имеем

$$
\begin{aligned}
I_{1}^{(\varepsilon)}\left[\zeta^{\varepsilon}\right] \equiv & \mu^{\varepsilon} \int_{K_{h}^{z} \cap \mathscr{M}^{(\varepsilon)}}\left(a \varepsilon^{\theta}\left|\nabla \zeta^{\varepsilon}\right|^{2}+\lambda \Phi_{m}\left|\zeta^{\varepsilon}\right|^{2}\right) d x \\
& +\mu^{\varepsilon} \int_{K_{h}^{z} \cap \mathscr{F}(\varepsilon)}\left(\left|\nabla \zeta^{\varepsilon}\right|^{2}+h^{-2-\gamma}\left|\zeta^{\varepsilon}\right|^{2}\right) d x \\
\leqslant & 2 a \varepsilon^{\theta} \mu^{\varepsilon} \int_{K_{h}^{z} \cap \partial \mathscr{M}^{(\varepsilon)}}\left|\frac{\partial \vartheta^{\varepsilon}}{\partial \nu}\right|\left|\zeta^{\varepsilon}\right| d \sigma .
\end{aligned}
$$

Нетрудно показать, что для любой функции $v \in H^{1}(\Omega)$ справедливо неравенство

$$
\int_{K_{h}^{z} \cap \partial \mathscr{M}^{(\varepsilon)}}|v|^{2} d \sigma \leqslant C\left(\frac{1}{d_{\varepsilon}} \int_{K_{h}^{z} \cap \mathscr{F}(\varepsilon)}|v|^{2} d x+d_{\varepsilon} \int_{K_{h}^{z} \cap \mathscr{F}(\varepsilon)}|\nabla v|^{2} d x\right) .
$$

Тогда из (7.15), (7.16) получаем

$$
\begin{aligned}
I_{1}^{(\varepsilon)}\left[\zeta^{\varepsilon}\right] \leqslant & C \mu^{\varepsilon} \varepsilon^{\theta}\left(\sum_{i} \int_{K_{h}^{z} \cap \partial \mathscr{M}_{i}^{(\varepsilon)}}\left|\frac{\partial \vartheta^{\varepsilon}}{\partial \nu}\right|^{2} d \sigma\right)^{1 / 2}\left(\sum_{i} \int_{K_{h}^{z} \cap \partial \mathscr{M}_{i}^{(\varepsilon)}}\left|\zeta^{\varepsilon}\right|^{2} d \sigma\right)^{1 / 2} \\
\leqslant & C \mu^{\varepsilon} \varepsilon^{\theta}\left(\sum_{i} \int_{K_{h}^{z} \cap \partial \mathscr{M}_{i}^{(\varepsilon)}}\left|\frac{\partial \vartheta^{\varepsilon}}{\partial \nu}\right|^{2} d \sigma\right)^{1 / 2} \\
& \times\left(\frac{1}{d_{\varepsilon}} \int_{K_{h}^{z} \cap \mathscr{F}(\varepsilon)}\left|\zeta^{\varepsilon}\right|^{2} d x+d_{\varepsilon} \int_{K_{h}^{z} \cap \mathscr{F}(\varepsilon)}\left|\nabla \zeta^{\varepsilon}\right|^{2} d x\right)^{1 / 2}
\end{aligned}
$$

Для того чтобы оценить нормальную производную функции $\vartheta^{\varepsilon}(x)$, воспользуемся следующей леммой.

Лемма 7.1. Пусть функиия $\vartheta^{\varepsilon}$ определяется равенством (7.12), әде $W^{\varepsilon}-$ решение задачи (7.7), (7.8). Тогда

$$
\left|\frac{\partial \vartheta^{\varepsilon}}{\partial \nu}\right| \leqslant C(\lambda) \varepsilon^{-\theta / 2}, \quad x \in \partial \mathscr{M}_{i}^{(\varepsilon)} .
$$

ДокАЗАТЕЛЬСтво. Для простоты будем предполагать, что $\mathscr{M}_{i}^{(\varepsilon)}=\left\{x \in \mathbb{R}^{3}\right.$ : $\left.0<x_{k}<\left(\varepsilon-d_{\varepsilon}\right)\right\}$. Из $(7.7),(7.8)$ следует что $\vartheta^{\varepsilon}(x)$ является решением краевой задачи

$$
\begin{gathered}
\Delta \vartheta^{\varepsilon}-\lambda \Phi_{m} \widetilde{\beta}_{\varepsilon} \vartheta^{\varepsilon}=0, \quad x \in \mathscr{M}_{i}^{(\varepsilon)}, \\
\vartheta^{\varepsilon}=1, \quad x \in \partial \mathscr{M}_{i}^{(\varepsilon)}
\end{gathered}
$$

где $\widetilde{\beta}^{\varepsilon}=a^{-1} \varepsilon^{-\theta}$. Рассмотрим функцию

$$
v^{\varepsilon}=\exp \left(-\sqrt{\lambda \Phi_{m} \widetilde{\beta}_{\varepsilon}} x_{1}\right) .
$$

Очевидно, что $v^{\varepsilon}(x)$ удовлетворяет уравнению $(7.19)$ и $v^{\varepsilon}(x)=1$ на грани $\left\{x_{1}=0\right\}$. Тогда в соответствии с принципом максимума функция $\vartheta^{\varepsilon}(x)-v^{\varepsilon}(x)$ достигает положительньй максимум (или отрицательный минимум) на $\partial \mathscr{M}_{i}^{(\varepsilon)}$. Поскольку 
функция $\vartheta^{\varepsilon}(x)-v^{\varepsilon}(x)$ равна нулю на грани $\left\{x_{1}=0\right\}$ и положительна на других гранях куба $\mathscr{M}_{i}^{(\varepsilon)}$, мы имеем

$$
\vartheta^{\varepsilon}-v^{\varepsilon} \geqslant 0, \quad x \in \overline{\mathscr{M}}_{i}^{(\varepsilon)} .
$$

С другой стороны, $\vartheta^{\varepsilon} \leqslant 1$ в $\overline{\mathscr{M}}_{i}^{(\varepsilon)}$. Тогда из $(7.22)$ следует, что

$$
1-v^{\varepsilon} \geqslant 1-\vartheta^{\varepsilon} \geqslant 0, \quad x \in \overline{\mathscr{M}}_{i}^{(\varepsilon)},
$$

и, поскольку $v^{\varepsilon}\left(0, x_{2}, x_{3}\right)=\vartheta^{\varepsilon}\left(0, x_{2}, x_{3}\right)=1$, мы получаем

$$
\frac{v^{\varepsilon}\left(0, x_{2}, x_{3}\right)-v^{\varepsilon}\left(\delta, x_{2}, x_{3}\right)}{\delta} \geqslant \frac{\vartheta^{\varepsilon}\left(0, x_{2}, x_{3}\right)-\vartheta^{\varepsilon}\left(\delta, x_{2}, x_{3}\right)}{\delta} \geqslant 0, \quad \delta>0 .
$$

Переходя к пределу в (7.24) при $\delta \rightarrow 0$, мы получаем

$$
0 \leqslant \frac{\partial \vartheta^{\varepsilon}}{\partial \nu} \leqslant \frac{\partial v^{\varepsilon}}{\partial \nu}, \quad x \in\left\{x_{1}=0\right\}
$$

причем

$$
\left.\frac{\partial v^{\varepsilon}}{\partial \nu}\right|_{x_{1}=0}=\sqrt{\frac{\lambda}{a}} \varepsilon^{-\theta / 2}
$$

Ясно, что нормальная производная $\vartheta^{\varepsilon}$ на других гранях $\mathscr{M}_{i}^{(\varepsilon)}$ оценивается так же. Лемма 7.1 доказана.

Из (7.18) теперь имеем

$$
\sum_{i} \int_{K_{h}^{z} \cap \partial \mathscr{M}_{i}^{(\varepsilon)}}\left|\frac{\partial \vartheta^{\varepsilon}}{\partial \nu}\right|^{2} d \sigma \leqslant C \frac{h^{3}}{\varepsilon^{\theta+1}} .
$$

Тогда, используя (7.1), (7.4) и (7.17), получаем

$$
\begin{aligned}
I_{1}^{(\varepsilon)}\left[\zeta^{\varepsilon}\right] \leqslant & C h^{3 / 2} \mu_{\varepsilon} \varepsilon^{(\theta-1) / 2}\left(d_{\varepsilon}\right)^{-1 / 2} \\
& \times\left(\int_{K_{h}^{z} \cap \mathscr{F}(\varepsilon)}\left|\zeta^{\varepsilon}\right|^{2} d x+d_{\varepsilon}^{2} \int_{K_{h}^{z} \cap \mathscr{F}(\varepsilon)}\left|\nabla \zeta^{\varepsilon}\right|^{2} d x\right)^{1 / 2} \\
\leqslant & C h^{5 / 2+\gamma / 2}\left(\mu_{\varepsilon}\right)^{1 / 2} \varepsilon^{(\theta-1) / 2}\left(d_{\varepsilon}\right)^{-1 / 2}\left(I_{1}^{(\varepsilon)}\left[\zeta^{\varepsilon}\right]\right)^{1 / 2} \\
\leqslant & C h^{5 / 2+\gamma / 2}\left(I_{1}^{(\varepsilon)}\left[\zeta^{\varepsilon}\right]\right)^{1 / 2} .
\end{aligned}
$$

Это означает, что функция $\zeta^{\varepsilon}(x)$ вносит исчезающий вклад в функционал (7.10), т.e.

$$
b_{\lambda}^{\varepsilon, h}(z)=\mu^{\varepsilon} \int_{K_{h}^{z}}\left(a \varepsilon^{\theta}\left|\nabla \vartheta^{\varepsilon}\right|^{2}+\lambda \Phi_{m}\left|\vartheta^{\varepsilon}\right|^{2}\right) \mathbb{I}_{m}^{\varepsilon}(x) d x+o\left(h^{\varepsilon}\right)
$$

при $h \rightarrow 0$ и достаточно малых $\varepsilon$. Таким образом, в силу $(7.7),(7.8)$ и $(7.27)$ получаем

$$
\lim _{h \rightarrow 0} h^{-3} \varlimsup_{\varepsilon \rightarrow 0} b_{\lambda}^{\varepsilon, h}(z)=\lim _{h \rightarrow 0} h^{-3} \underline{\lim }_{\varepsilon \rightarrow 0} b_{\lambda}^{\varepsilon, h}(z)=\lim _{\varepsilon \rightarrow 0} \mu^{\varepsilon} \lambda \Phi_{m} \int_{\mathscr{M}} W^{\varepsilon}(x) d x
$$

при условии, что послений предел сушествует.

Остается только вычислить асимптотику интеграла в (7.28). 
Лемма 7.2. Пусть $W^{\varepsilon}(x)$ - решение краевой задачи (7.7), (7.8). Тогда

$$
\int_{\mathscr{M}} W^{\varepsilon}(x) d x=\frac{6}{\sqrt{\lambda}} \frac{1}{\sqrt{\Phi_{m} \beta_{\varepsilon}}}(1+o(1))
$$

npu $\varepsilon \rightarrow 0$.

ДокАЗАТЕЛЬСТво. В кубе $\mathscr{M}$ рассмотрим функции

$$
V_{\varepsilon j}^{ \pm}(x)=\exp \left( \pm \sqrt{\lambda \Phi_{m} \beta_{\varepsilon}}\left(x_{j} \mp \frac{1}{2}\right)\right), \quad j=1,2,3,
$$

и

$$
U^{\varepsilon}(x)=\sum_{j=1}^{3}\left(V_{\varepsilon j}^{+}(x)+V_{\varepsilon j}^{-}(x)\right) .
$$

Они удовлетворяют $(7.7)$, причем $V_{\varepsilon j}^{ \pm}(x) \leqslant 1$ при $x \in \partial \mathscr{M}$, а $U^{\varepsilon}(x) \geqslant 1$ при $x \in \partial \mathscr{M}$. Тогда в силу принципа максимума

$$
V_{\varepsilon j}^{ \pm}(x) \leqslant W^{\varepsilon}(x) \leqslant U^{\varepsilon}(x) .
$$

Введем в рассмотрение множества

$$
\begin{aligned}
M_{\varepsilon j}^{ \pm}=\{x & \in \mathscr{M}:-\frac{1}{2} \leqslant \mp x_{j} \leqslant \frac{1}{2}+\left(\beta_{\varepsilon}\right)^{-1 / 4}, \\
& \left.-\frac{1}{2}+\left(\beta_{\varepsilon}\right)^{-1 / 4} \leqslant x_{k} \leqslant \frac{1}{2}-\left(\beta_{\varepsilon}\right)^{-1 / 4}, j \neq k\right\} .
\end{aligned}
$$

Ясно, что $M_{\varepsilon j}^{ \pm} \cap M_{\varepsilon k}^{ \pm}=\varnothing$ при $j \neq k$ и достаточно малых $\varepsilon$. Тогда из (7.32) вытекает, что

$$
\sum_{j=1}^{3}\left(\int_{M_{\varepsilon j}^{+}} V_{\varepsilon j}^{+}(x) d x+\int_{M_{\varepsilon j}^{-}} V_{\varepsilon j}^{-}(x) d x\right) \leqslant \int_{\mathscr{M}} W^{\varepsilon}(x) d x \leqslant \int_{\mathscr{M}} U^{\varepsilon}(x) d x .
$$

Легко видеть, что

$$
\sum_{j=1}^{3}\left(\int_{M_{\varepsilon j}^{+}} V_{\varepsilon j}^{+}(x) d x+\int_{M_{\varepsilon j}^{-}} V_{\varepsilon j}^{-}(x) d x\right)=\frac{6}{\sqrt{\lambda \Phi_{m} \beta_{\varepsilon}}}\left(1+o\left(\beta_{\varepsilon}^{-1 / 4}\right)\right)
$$

и

$$
\int_{\mathscr{M}} U^{\varepsilon}(x) d x=\frac{6}{\sqrt{\lambda \Phi_{m} \beta_{\varepsilon}}}\left(1+o\left(\beta_{\varepsilon}^{-1 / 4}\right)\right) .
$$

Утвеждение леммы следует из (7.33), (7.34), и (7.35). Лемма 7.2 доказана.

Таким образом, в силу (7.28), (7.29)

$$
b(x, \lambda)=b(\lambda)=\frac{2 \sqrt{a \Phi_{m}}}{d} \sqrt{\lambda}
$$

и

$$
B(x, t)=B(t)=\frac{\sqrt{a \Phi_{m}}}{\pi i d} \int_{\sigma-i \infty}^{\sigma+i \infty} e^{\lambda t} \frac{d \lambda}{\sqrt{\lambda}}=\frac{2 \sqrt{a \Phi_{m}}}{\sqrt{\pi} d} \frac{1}{\sqrt{t}} .
$$

Поэтому условие (C.4) выполнено и, значит, доказан следующий результат. 
TEорема 7.1. Пусть $\Omega=\mathscr{F}^{(\varepsilon)} \cup \mathscr{M}^{(\varepsilon)} \cup \partial \mathscr{M}^{(\varepsilon)}$ - ограниченная область в $\mathbb{R}^{3}$ с кусочно-гладкой границей $\partial \Omega$. Предположим, что система трещин $\mathscr{F}(\varepsilon)$ распределена в тонких ортогональных слоях толщины $d_{\varepsilon}, d_{\varepsilon}=d \varepsilon^{\theta / 2}, d>0$, $\theta>2$, а матрица $\mathscr{M}^{(\varepsilon)}$ - множество кубов, периодически, с периодом $\varepsilon$, распределенных в $\Omega$.

Тогда для любого $t \in(0, T)$ решение $u^{\varepsilon}(x, t)$ начально-краевой задачи задачи (1.4)-(1.6) с $A^{\varepsilon}(x)=a^{\varepsilon}(x) I$, әде $I$ - единичная матрица, а $a^{\varepsilon}(x)=a \varepsilon^{\theta} \cdot \mathbb{I}_{m}^{\varepsilon}(x)+$ $1 \cdot \mathbb{I}_{f}^{\varepsilon}(x), D_{\mathscr{F}(\varepsilon)}$-сходится к решению $и(x, t)$ следующей задачи

$$
\begin{gathered}
\Phi_{f} u_{t}-\frac{2}{3} \Delta u+\frac{2 \sqrt{a \Phi_{m}}}{\sqrt{\pi} d} \int_{0}^{t} \frac{u_{t}(x, \tau)}{\sqrt{t-\tau}} d \tau=f(x), \quad x \in \Omega_{T}, \\
\frac{\partial u}{\partial \nu}(x, t)=0, \quad x \in \partial \Omega, \quad t \in(0, T), \\
u(x, 0)=0, \quad x \in \Omega .
\end{gathered}
$$

Причем, так как и $(x, t)-$ гладкая в $\Omega$ функиия, имеем

$$
\lim _{\varepsilon \rightarrow 0} \frac{1}{\operatorname{meas} \mathscr{F}(\varepsilon)}\left\|u^{\varepsilon}-u\right\|_{\mathscr{F}(\varepsilon)}^{2}=0
$$

ЗАмЕчАнИЕ 7.1. Соотношения (7.1), (7.2) определяют критическую толщину трещин в случае, когда обмен между матрицей и трещинами нельзя не учитывать. Если коэффициент диффузии $a^{\varepsilon}(x)$ по-прежнему определяется соотншением $(7.2)$, но толщина $d_{\varepsilon}$ много больше чем $\sqrt{a^{\varepsilon}(x)}$ при $x \in \mathscr{M}^{\varepsilon}$, тогда процесс переноса определяется только системой трешин. А именно, для любого $t \in(0, T)$ решение $u^{\varepsilon}(x, t)$ сходится в смысле $(7.36)$ к решению $u(x, t)$ краевой задачи

$$
\begin{gathered}
\Phi_{f} u_{t}-\frac{2}{3} \Delta u=f(x), \quad x \in \Omega_{T}, \\
\frac{\partial u}{\partial \nu}(x, t)=0, \quad x \in \partial \Omega, t \in(0, T) ; \\
u(x, 0)=0, \quad x \in \Omega .
\end{gathered}
$$

Заметим, что такой же усредненный тензор для рассматриваемой структуры множества $\mathscr{F}^{(\varepsilon)}$ (сотовая или альвеолярная структура) получен в [25] другими методами.

В заключение авторы выражают благодарность профессорам А. Буржа, М.Б. Панфилову и Е.Я. Хруслову за полезные обсуждения и внимание к работе.

\section{Список литературы}

1. Санчес-Паленсия Э. Неоднородные среды и теория колебаний. М.: Мир, 1984.

2. Bensoussan A., Lions J.-L., Papanicolau G. Asymptotic analysis for periodic structures. Amsterdam: North-Holland, 1978.

3. Бахвалов Н. С., Панасенко Г. П. Осреднение процессов в периодических средах. М.: Наука, 1984.

4. Жиков В.В., Козлов С. М., Олейник О.А. Усреднение дифференциальных операторов. М.: Физматлит, 1993.

5. Марченко B. A., Хруслов E. Я. Краевые задачи в областях с мелко-зернистой границей. Киев: Наукова думка, 1974.

6. Баренблатт Г.И., Желтов Ю. П., Кочина И. Н. Основы теории течения однородной жидкости в пористых средах // Межвуз. тематич. сб. Прикл. матем. и мех. 1960. T. 24. C. $852-854$. 
7. Arbogast T., Douglas J., Hornung U. Derivation of the double porosity model of single phase flow via homogenization theory // SIAM J. Appl. Math. 1990. V. 21. P. 823-826.

8. Bourgeat A., Goncharenko M., Panfilov M., Pankratov L. A general double porosity model // C. R. Acad. Sci. Paris. Sér. IIb. 1999. V. 327. P. 1245-1250.

9. Bourgeat A., Luckhaus S., Mikelic A. Convergence of the homogenization process for a double porosity model of immiscible two phase flow // SIAM J. Appl. Math. 1996. V. 27. P. $1520-1543$.

10. Сандраков Г. В. Осреднение параболических уравнений с контрастньми коэффициентами // Изв. РАН. Сер. матем. 1999. Т. 63. № 4. С. 17-224.

11. Hornung U. (ed.) Homogenization and porous media. New York: Springer-Verlag, 1997.

12. Жиков В. В. Связность и усреднение. Примеры фрактальной проводимости // Матем. сб. 1996. Т. 187. № 8. С. 3-40.

13. ЖКиков В. В. О весовых соболевских пространствах // Матем. сб. 1998. Т. 189. № 8. C. $27-58$

14. Жиков В. В. Об одном расширении и применении метода двухмасштабной сходимости // Матем. сб. 2000. Т. 191. № 7. С. 31-72.

15. Auriault J. L. Effective macroscopic description for heat conduction in periodic composites // Int. J. Heat Mass Transfer. 1983. V. 26. №6. P. 861-869.

16. Bourgeat A., Mikelic A., Piatnitski A. Modèle de double porosité aléatoire // C. R. Acad. Sci. Paris. Sér. I. 1998. V. 327. P. 99-104

17. Ладыженская О.А., Солонников В. А., Уральцева Н. Н. Линейныеи квазилинейные уравнения параболического типа. М.: Наука, 1967.

18. Свищева E. B. Асимптотическое поведение решений решений второй краевой задачи в областях убывающего объема // Теория операторов и субгармонические функции. Киев: Наукова думка, 1991. С. 126-134.

19. Фенченко B.M., Хруслов E. Я. Асимптотическое поведение решений дифференциальных уравнений в частных производных с сильно осциллирующей матрицей коэффициентов, которая не удовлетворяет условию равномерной ограниченности // Докл. АН УССР. Сер. А. 1981. № 4. С. 23-27.

20. Khruslov E. Ya. Homogenized models of composite media // Composite media and homogenization theory / ed. G. Dal Maso, G.F. Dell'Antonio. Boston: Birkhäuser, 1991. P. 159-182. (Progr. Nonlinear Differential Equations. Appl. V. 5.)

21. Хруслов $E$. $Я$. Асимптотическое поведение решений второй краевой задачи при измельчении границы области // Матем. сб. 1978. Т. 106. № 4. С. 603-621.

22. Стейн И. Сингулярные интегралы и дифференциальные свойства функций. М.: Мир, 1973.

23. Boutet de Monvel L., Khruslov E. Ya. Averaging of the diffusion equation on Riemannian manifolds of complex microstructure // Trans. Moscow Math. Soc. 1997. P. 138-161.

24. Фенченко B. Н. Асимптотика решений дифференциальных уравнений в частных производных с сильно осциллирующей и неограниченной матрицей коэффициентов // Препринт. №30. Харьков: ФТИНТ АН УССР, 1980.

25. Cioranescu D., Saint Jean Paulin J. Homogenization of reticulated structures. New York: Springer-Verlag, 1999. (Appl. Math. Sci. V. 136.)

Физико-технический институт

низких температур им. Б.И. Веркина,

г. Харьков

E-mail: pankratov@ilt.kharkov.ua, vrybalko@ilt.kharkov.ua
Поступила в редакцию 18.12 .2001 и 14.08 .2002 